\title{
Aspects of the derivative coupling model in four dimensions
}

\author{
Andreas Aste ${ }^{1,2, a}$ \\ ${ }^{1}$ Department of Physics, University of Basel, 4056 Basel, Switzerland \\ 2 Paul Scherrer Institute, 5232 Villigen PSI, Switzerland
}

Received: 9 July 2013 / Accepted: 5 December 2013 / Published online: 23 January 2014

(C) The Author(s) 2014. This article is published with open access at Springerlink.com

\begin{abstract}
A concise discussion of a $3+1$-dimensional derivative coupling model, in which a massive Dirac field couples to the four-gradient of a massless scalar field, is given in order to elucidate the role of different concepts in quantum field theory like the regularization of quantum fields as operator-valued distributions, correlation distributions, locality, causality, and field operator gauge transformations.
\end{abstract}

\section{Introduction}

Quantum field theory (QFT) is plagued by many conceptual problems. It has hitherto been impossible to prove the existence of a non-trivial QFT in four space-time dimensions. For example, it is notoriously difficult for perturbative QFTs to establish convergence of expansions of the $S$-matrix and related observable quantities. Despite this fact, perturbative QFT has been very successful in predicting measurable quantities in elementary particle physics. On the perturbative level, infrared and ultraviolet divergences can be handled by several mathematical tricks and tools. Whereas ultraviolet divergences are rather related to the short distance behavior of a QFT, integrals over infinite space-time result in some sort of infrared difficulties when massless fields are involved, depending on the approach that was chosen to formulate the theory.

As a general remark, one may say that QFT on unquantized space-time can be considered as some sort of operatorvalued distribution theory, which respects basic inputs coming from symmetry considerations which normally include the Poincare symmetry group $\mathcal{P}_{+}^{\uparrow}$ as the semidirect product of the abelian group of time-space translations $T_{1,3}$ and the restricted Lorentz group $\mathrm{SO}^{+}(1,3)$, or, to be more precise, the covering group $\overline{\mathcal{P}}_{+}^{\uparrow}=T_{1,3} \rtimes S L(2, \mathbb{C})[1]$.

Even the definition of a particle in non-gravitating flat space-time becomes a non-trivial task when charged particles

\footnotetext{
a e-mail: andreas.aste@unibas.ch
}

coupling to massless gauge fields become involved. Based on the classical analysis of Wigner on the unitary representations of the Poincaré group, a one-particle state is an element of an irreducible representation space of the double cover of the Poincare group in a physical Hilbert space, i.e. some irreducible representations should occur in the discrete spectrum of the mass-squared operator $M^{2}=P_{\mu} P^{\mu}$ of a QFT describing particles [2]. However, objects like the electron are accompanied by a long range field which leads an independent life at infinite spatial distance, to give an intuitive picture. It has been shown in [3] that a discrete eigenvalue of $M^{2}$ is absent for states with an electric charge as a direct consequence of Gauss' law, and one finds that the Lorentz symmetry is not implementable in a sector of states with nonvanishing electric charge. Such problems are related to the fact that the Poincaré symmetry is an overidealization related to global considerations of infinite flat space-time, however, physical measurements have a local character.

In this paper, we follow a shut-up and calculate approach, in order to point out the fact that many aspects of QFT are still poorly understood and to demonstrate the mathematical apparatus which is treated very often on a fairly phenomenological level. The derivative coupling model, which serves thereby as a trivial, but stunning example for this fact, will be discussed in two different versions.

\section{The classical derivative coupling model}

As a starting point for the derivative coupling model discussed in this paper, one may consider the equations of motion of the coupled Maxwell-Dirac system where a massive spin- $1 / 2$ field $\psi$ couples to a massless abelian spin-1 gauge field $A_{\mu}$ in the Feynman gauge,

$$
\begin{aligned}
& \left(i \gamma_{\mu} \partial^{\mu}-m\right) \psi(x)=e A^{\mu}(x) \gamma_{\mu} \psi(x), \\
& \square A_{\mu}(x)=j_{\mu}(x)=e \bar{\psi}(x) \gamma_{\mu} \psi(x),
\end{aligned}
$$

where, e.g., a coupling constant $e<0$ would relate to a field $\psi$ describing negatively charged objects like electrons as par- 
ticles and the positively charged positrons as anti-particles. $\gamma^{0}, \ldots, \gamma^{3}$ are Dirac matrices fulfilling the standard anticommutation relations. Replacing $A_{\mu}$ by the four-gradient of a massless, neutral scalar field $\varphi$ [4] and, in order to clearly distinguish the two theories from a notational point of view, the electric coupling constant $e$ by a coupling constant $g$ leads to the defining equations of the derivative coupling model

$$
\begin{aligned}
& \left(i \gamma_{\mu} \partial^{\mu}-m\right) \psi(x)=g \partial^{\mu} \varphi(x) \gamma_{\mu} \psi(x), \\
& \square \varphi(x)=\partial^{\mu} j_{\mu}(x)=0 .
\end{aligned}
$$

These equations can be derived from the Lagrangian

$$
\begin{aligned}
\mathcal{L} & =i \bar{\psi} \gamma^{\mu} \partial_{\mu} \psi-m \bar{\psi} \psi+\frac{1}{2} \partial_{\mu} \varphi \partial^{\mu} \varphi-g \partial^{\mu} \varphi \bar{\psi} \gamma_{\mu} \psi \\
& =\mathcal{L}_{\psi}^{0}+\mathcal{L}_{\varphi}^{0}+\mathcal{L}_{\text {int }}
\end{aligned}
$$

with

$\mathcal{L}_{\text {int }}=-g \partial^{\mu} \varphi \bar{\psi} \gamma_{\mu} \psi$.

In classical field theory, a solution of Eqs. (3) and (4) is readily found

$\psi(x)=\mathrm{e}^{-i g \varphi(x)} \psi_{0}(x)$

with free fields $\varphi(x)$ and $\psi_{0}(x)$ satisfying

$\square \varphi(x)=0, \quad\left(i \gamma_{\mu} \partial^{\mu}-m\right) \psi_{0}(x)=0$,

since one has

$$
\begin{aligned}
\left(i \gamma_{\mu} \partial^{\mu}-m\right) \psi & =i \gamma_{\mu} \partial^{\mu}\left(\psi_{0} \mathrm{e}^{-i g \varphi}\right)-m \psi \\
& =i \gamma_{\mu} \mathrm{e}^{-i g \varphi(x)} \partial^{\mu} \psi_{0}-m \psi+g \partial^{\mu} \varphi \gamma_{\mu} \psi \\
& =g \partial^{\mu} \varphi \gamma_{\mu} \psi
\end{aligned}
$$

Leaving the classical level, it may be argued that the interacting Dirac field is 'dressed' in some sense by excitations of the massless bosonic field. However, since quantum fields are operator-valued distributions, products or exponentials of such objects are not defined in general and require a thorough discussion. Field products are unavoidable for the construction of observables, since neither the Dirac field nor the vector potential corresponds to observable quantities. Still, it seems evident that the derivative coupling model is physically trivial since the Dirac field couples to a pure gauge. The model itself is invariant under gauge transformations,

$\psi^{\prime}(x)=\mathrm{e}^{-i g \chi(x)} \psi(x), \quad \varphi^{\prime}(x)=\varphi(x)+\chi(x)$,

where again $\square \chi(x)=0$, and a mass term for the scalar field $\varphi$ could be included in the model, but this option will not be considered in this paper.

\section{Preliminaries and conventions}

\subsection{The free scalar field}

In order to provide a well-defined setting for the forthcoming discussion of the derivative coupling model on a quantum field theoretical level, we discuss some basic properties and definitions concerning the free, i.e. non-interacting scalar field describing a neutral or charged spin-0 particle of mass $M$ in $(3+1)$ space-time dimensions. Such a discussion may appear as overkill, but it is not. Scalar bosonic fields may be represented according to

$$
\begin{aligned}
\varphi(x)= & \varphi^{-}(x)+\varphi^{+}(x)=\frac{1}{(2 \pi)^{3 / 2}} \int \frac{\mathrm{d}^{3} k}{\sqrt{2 k^{0}}} \\
& \times\left[a(\boldsymbol{k}) \mathrm{e}^{-i k x}+a^{\dagger}(\boldsymbol{k}) \mathrm{e}^{+i k x}\right] \quad(\text { neutral }), \\
\varphi_{c}(x)= & \varphi_{c}^{-}(x)+\varphi_{c}^{+}(x)=\frac{1}{(2 \pi)^{3 / 2}} \int \frac{\mathrm{d}^{3} k}{\sqrt{2 k^{0}}} \\
& \times\left[a(\boldsymbol{k}) \mathrm{e}^{-i k x}+b^{\dagger}(\boldsymbol{k}) \mathrm{e}^{+i k x}\right] \quad \text { (charged), }
\end{aligned}
$$

where $k x=k_{\mu} x^{\mu}=k^{0} x^{0}-\boldsymbol{k} \cdot \boldsymbol{x}, k^{0} \stackrel{!}{=} E=\sqrt{\boldsymbol{k}^{2}+M^{2}}>$ $0, \pm$ denotes the positive and negative frequency parts of the fields, and † 'hermitian conjugation'. The non-vanishing distributional commutator relations for the destruction and creation field operators in the above Fourier decomposition are

$\left[a(\boldsymbol{k}), a^{\dagger}\left(\boldsymbol{k}^{\prime}\right)\right]=\left[b(\boldsymbol{k}), b^{\dagger}\left(\boldsymbol{k}^{\prime}\right)\right]=\delta^{(3)}\left(\boldsymbol{k}-\boldsymbol{k}^{\prime}\right)$,

otherwise

$$
\begin{aligned}
{\left[a(\boldsymbol{k}), a\left(\boldsymbol{k}^{\prime}\right)\right] } & =\left[b(\boldsymbol{k}), b\left(\boldsymbol{k}^{\prime}\right)\right] \\
& =\left[a^{\dagger}(\boldsymbol{k}), a^{\dagger}\left(\boldsymbol{k}^{\prime}\right)\right]=\left[b^{\dagger}(\boldsymbol{k}), b^{\dagger}\left(\boldsymbol{k}^{\prime}\right)\right]=0
\end{aligned}
$$

and

$$
\begin{aligned}
{\left[a(\boldsymbol{k}), b\left(\boldsymbol{k}^{\prime}\right)\right] } & =\left[a(\boldsymbol{k}), b^{\dagger}\left(\boldsymbol{k}^{\prime}\right)\right] \\
& =\left[a^{\dagger}(\boldsymbol{k}), b\left(\boldsymbol{k}^{\prime}\right)\right]=\left[a^{\dagger}(\boldsymbol{k}), b^{\dagger}\left(\boldsymbol{k}^{\prime}\right)\right]=0
\end{aligned}
$$

holds. The destruction (or 'annihilation', or 'absorption') operators act on the non-degenerate vacuum $|0\rangle$ according to

$a(\boldsymbol{k})|0\rangle=b(\boldsymbol{k})|0\rangle=0$ for all $k \in \mathbb{R}^{3}$.

It is crucial to require the existence of a state $|0\rangle$ which is annihilated by all the $a(\boldsymbol{k})$ and $b(\boldsymbol{k})$, since otherwise there would be many inequivalent irreducible Hilbert space representations of the algebraic relations given by Eqs. (13)-(15), and Eq. (16) selects the one in Fock space where the $a(\boldsymbol{k})$ and $b(\boldsymbol{k})$ can be interpreted as destruction and the $a^{\dagger}(\boldsymbol{k})$ and $b^{\dagger}(\boldsymbol{k})$ as creation (or 'emission') operators. 
Single-particle wave functions in momentum space $\Psi_{1}(\boldsymbol{k})$, $\Psi_{2}(\boldsymbol{k})$ are

$$
\begin{aligned}
& \left|\Psi_{1}\right\rangle=\int \mathrm{d}^{3} k \Psi_{1}(\boldsymbol{k}) a^{\dagger}(\boldsymbol{k})|0\rangle, \\
& \left|\Psi_{2}\right\rangle=\int \mathrm{d}^{3} k^{\prime} \Psi_{2}\left(\boldsymbol{k}^{\prime}\right) a^{\dagger}\left(\boldsymbol{k}^{\prime}\right)|0\rangle,
\end{aligned}
$$

their scalar product becomes, from a formal calculation exploiting the commutation relations above,

$$
\begin{aligned}
& \left\langle\Psi_{1} \mid \Psi_{2}\right\rangle \\
& =\int \mathrm{d}^{3} k \mathrm{~d}^{3} k^{\prime} \Psi_{1}(\boldsymbol{k})^{*} \Psi_{2}\left(\boldsymbol{k}^{\prime}\right)\left\langle 0 \mid a(\boldsymbol{k}) a^{\dagger}\left(\boldsymbol{k}^{\prime}\right) 0\right\rangle \\
& =\int \mathrm{d}^{3} k \mathrm{~d}^{3} k^{\prime} \Psi_{1}(\boldsymbol{k})^{*} \Psi_{2}\left(\boldsymbol{k}^{\prime}\right)\left\langle 0\left|\left[\delta^{(3)}\left(\boldsymbol{k}-\boldsymbol{k}^{\prime}\right)+a^{\dagger}\left(\boldsymbol{k}^{\prime}\right) a(\boldsymbol{k})\right]\right| 0\right\rangle \\
& =\int \mathrm{d}^{3} k \Psi_{1}(\boldsymbol{k})^{*} \Psi_{2}(\boldsymbol{k}) .
\end{aligned}
$$

This scalar product can be written in a manifestly covariant form by using differently normalized creation and destruction operators fulfilling

$\left[a(\boldsymbol{k}), a^{\dagger}\left(\boldsymbol{k}^{\prime}\right)\right]=\left[b(\boldsymbol{k}), b^{\dagger}\left(\boldsymbol{k}^{\prime}\right)\right]=(2 \pi)^{3 / 2}\left(2 k^{0}\right)^{1 / 2} \delta^{(3)}\left(\boldsymbol{k}-\boldsymbol{k}^{\prime}\right)$.

\subsection{Quantum fields as operator-valued distributions}

It is crucial to note that $\varphi(x)$ and $\varphi_{c}(x)$ are operator-valued distributions, i.e. only smeared out fields like

$\varphi(g)=\int \mathrm{d}^{4} x \varphi(x) g(x)$,

where $g$ is a test function is some suitable test function space $\mathcal{T}\left(\mathbb{R}^{4}\right)$, are operators in the quantum mechanical sense on the Hilbert-Fock space of free particles, i.e. linear operators defined on a dense subset of the Hilbert space which are not necessarily bounded $[5,6]$. The same observation applies in momentum space, i.e.

$a^{\dagger}(\hat{g})=\int \mathrm{d}^{4} k a^{\dagger}(k) \hat{g}(k)$

creates a physical, i.e. normalizable Fock state, whereas $a^{\dagger}(\boldsymbol{k})|0\rangle$ is not a vector in Fock space, since no finite norm can be assigned to such an object due to Eq. (13). In fact, smearing field operators of a four-dimensional field theory in three dimensions as anticipated in Eq. (17) does not work, in general, in the case of interacting fields.

It is common usage in QFT in $n$ space-time dimensions to work with test functions which are elements of the Schwartz space of rapidly decreasing functions $\mathcal{S}\left(\mathbb{R}^{n}\right)$. This space is obtained by considering complex-valued $p$-times continuously differentiable functions in $C^{p}\left(\mathbb{R}^{n}\right)$ equipped with the norms

$$
\begin{array}{r}
\|f\|_{p}=\sup _{|\alpha| \leq p} \sup _{x \in \mathbb{R}^{n}}(1+\|x\|)^{p}\left|D^{\alpha} f(x)\right|, \\
x=\left(x^{1}, \ldots x^{n}\right), \quad\|x\|=\sqrt{\sum_{i=1}^{n}\left(x^{i}\right)^{2},}
\end{array}
$$

with multi-indices $\alpha=\left(\alpha_{1}, \ldots \alpha_{n}\right) \in \mathbb{N}_{0}^{n}$ and differential operators

$D^{\alpha}=\frac{\partial^{\alpha_{1}}}{\partial x^{\alpha_{1}}} \cdots \frac{\partial^{\alpha_{n}}}{\partial x^{\alpha_{n}}}, \quad$ where $|\alpha|=\alpha_{1}+\cdots+\alpha_{n}$,

defining thereby complete normed function spaces

$\bar{S}_{p}\left(\mathbb{R}^{n}\right)=\left\{f \in C^{p}\left(\mathbb{R}^{n}\right) \mid\|f\|_{p}<\infty\right\}$.

The Schwartz space $\mathcal{S}\left(\mathbb{R}^{n}\right)$ is then defined as the space of infinitely differentiable functions of rapid decrease

$\mathcal{S}\left(\mathbb{R}^{n}\right)=\bigcap_{p=0}^{\infty} \bar{S}_{p}\left(\mathbb{R}^{n}\right)$.

By a meaningful definition, a series of test functions $\left\{f_{v}\right\}_{\nu=0}^{\infty} \subset \mathcal{S}\left(\mathbb{R}^{n}\right)$ converges towards $f=0$ iff $\left\|f_{v}\right\|_{p} \stackrel{\nu \rightarrow \infty}{\rightarrow}$ 0 for all $p \in \mathbb{N}_{0}$. A typical example for a test function in $\mathcal{S}(\mathbb{R})$ is given by $g(x)=\mathrm{e}^{-x^{2}}$.

The space of tempered distributions $\mathcal{S}^{\prime}\left(\mathbb{R}^{n}\right)$ is the set of the continuous linear functionals on $\mathcal{S}\left(\mathbb{R}^{n}\right)$ according to

$d \in \mathcal{S}^{\prime}\left(\mathbb{R}^{n}\right) \Leftrightarrow d\left(f_{\nu}\right) \rightarrow 0 \quad$ for all $\left\{f_{\nu}\right\}_{\nu=0}^{\infty} \subset \mathcal{S}\left(\mathbb{R}^{n}\right)$

where $f_{v} \stackrel{v \rightarrow \infty}{\rightarrow} 0$. This definition of a tempered distribution becomes more intuitive if one realizes that such an object can be represented as the sum of derivatives of continuous functions of polynomial growth,

$$
\begin{aligned}
d \in & \mathcal{S}\left(\mathbb{R}^{n}\right) \Leftrightarrow d(f) \\
= & \sum_{0 \leq|\alpha| \leq s \in \mathbb{N}} \int \mathrm{d} x^{1} \cdots \mathrm{d} x^{n} \\
& \quad \times(-1)^{|\alpha|} F_{\alpha}\left(x^{1}, \ldots, x^{n}\right) D^{\alpha} f\left(x^{1}, \ldots, x^{n}\right),
\end{aligned}
$$

where $C\left(\mathbb{R}^{n}\right) \ni\left|F_{\alpha}(x)\right| \leq c_{\alpha}(1+\|x\|)^{j(\alpha)}$ for some $j(\alpha) \in$ $\mathbb{N}$ and $c_{\alpha} \in \mathbb{R}$. Formally, derivatives can be shifted by partial integration from test functions to distributions.

The true reason for using the Schwartz space in QFT is its convenient property that the Fourier transform acts on $\mathcal{S}\left(\mathbb{R}^{n}\right)$ as a unitary, bijective mapping, i.e. the Fourier transform of a smooth, rapidly decreasing function is again smooth and rapidly decreasing. This allows one to define the Fourier transform $\mathcal{F}$ of singular objects like the distributions in $\mathcal{S}^{\prime}\left(\mathbb{R}^{n}\right) . \hat{d}=\mathcal{F}(d)$ is defined so that for all $f \in \mathcal{S}\left(\mathbb{R}^{n}\right)$

$$
\mathcal{F}(d)(f)=\hat{d}(f)=d(\mathcal{F}(f))=d(\hat{f}),
$$


a definition which is often expressed by the purely formal expression involving a change in the order of integration

$$
\begin{aligned}
\hat{d}(f) & =\int_{\mathbb{R}^{n}} \mathrm{~d} x \hat{d}(x) f(x) \\
& =\frac{1}{(2 \pi)^{n / 2}} \int_{\mathbb{R}^{n}} \mathrm{~d} x \int_{\mathbb{R}^{n}} \mathrm{~d} k d(k) \mathrm{e}^{-i k x} f(x) \\
& =\int_{\mathbb{R}^{n}} \mathrm{~d} k d(k) \hat{f}(k) .
\end{aligned}
$$

Equivalently we have

$\hat{d}(\check{f})=d(\hat{\tilde{f}})=d(f)$.

This way, the Fourier transform also becomes a linear automorphism of $\mathcal{S}^{\prime}$

$\mathcal{F}\left(\mathcal{S}\left(\mathbb{R}^{n}\right)\right)=\mathcal{S}\left(\mathbb{R}^{n}\right), \quad \mathcal{F}\left(\mathcal{S}^{\prime}\left(\mathbb{R}^{n}\right)\right)=\mathcal{S}^{\prime}\left(\mathbb{R}^{n}\right)$.

Throughout this paper, the Fourier transform of a function on four-dimensional space-time will be defined according to the sign and symmetric normalization convention

$$
\begin{aligned}
\hat{\Phi}(k) & =\frac{1}{(2 \pi)^{2}} \int_{\mathbb{R}^{4}} \mathrm{~d}^{4} x \Phi(x) \mathrm{e}^{i k x} \\
& =\frac{1}{(2 \pi)^{2}} \int_{\mathbb{R}^{4}} \mathrm{~d}^{4} x \Phi(x) \mathrm{e}^{i k^{0} x^{0}-i k x},
\end{aligned}
$$

with $k x=k_{\mu} x^{\mu}=k_{0} x^{0}+k_{1} x^{1}+k_{2} x^{2}+k_{3} x^{3}=k^{0} x^{0}-$ $k^{1} x^{1}-k^{2} x^{2}-k^{3} x^{3}$.

An important subspace of distributions in $\mathcal{D}\left(\mathbb{R}^{n}\right) \subset$ $\mathcal{S}\left(\mathbb{R}^{n}\right)$ is spanned by the distributions of compact support. The dual space $\mathcal{D}^{\prime}\left(\mathbb{R}^{n}\right)$ of linear functionals on this space is more general than $\mathcal{S}^{\prime}\left(\mathbb{R}^{n}\right)$ and contains it. For the sake of brevity, topological aspects of $\mathcal{D}\left(\mathbb{R}^{n}\right)$ and $\mathcal{D}^{\prime}\left(\mathbb{R}^{n}\right)$ will not be discussed here. However, it is important to note that causality in QFT is often expressed by a relation of the form

$\left[O_{1}\left(g_{1}\right), O_{2}\left(g_{2}\right)\right]=0$ for $\operatorname{supp}\left(g_{1}\right) \sim \operatorname{supp}\left(g_{2}\right)$,

which expresses the fact that two local observables $O_{1}$ and $O_{2}$ depending as operator-valued distributions on test functions $g_{1}, g_{2} \in \mathcal{D}\left(\mathbb{R}^{n}\right) \subset \mathcal{S}\left(\mathbb{R}^{n}\right)$ commute whenever the compact supports of the test functions are space-like separated, i.e. when $\left(x_{1}-x_{2}\right)^{2}<0$ holds for all $x_{1} \in \operatorname{supp}\left(g_{1}\right)$ and $x_{2} \in \operatorname{supp}\left(g_{2}\right)$. One should note that the Fourier transforms $\hat{g_{1}}$ and $\hat{g_{2}}$ do not have a compact support for $g_{1}, g_{2} \neq 0$. The commutator, Eq. (33), may become an anti-commutator when fermionic fields are involved. However, such fields are elements of a field algebra and not of an algebra of observables, but they often serve as building blocks for the construction of observables.
In Appendix A, a well-known but indispensable set of relations needed for the manipulation of distributions is given for the reader who only has enjoyed a cursory formal introduction to the theory.

\subsection{Correlation distributions}

From the above algebraic relations represented by free fields on a Fock space $\mathcal{F}$ one constructs the scalar Feynman propagator as distributional time-ordered vacuum expectation values

$\Delta_{F}(x-y)=-i\langle 0| T\left(\varphi_{c}(x) \varphi_{c}^{\dagger}(y)|0\rangle\right.$,

where translational invariance implies

$\Delta_{F}(x)=-i\langle 0| T\left(\varphi_{c}(x) \varphi_{c}^{\dagger}(0)|0\rangle\right.$

or

$\Delta_{F}(x)=-i\langle 0| T(\varphi(x) \varphi(0)|0\rangle$,

for neutral fields. The wave equation holds in a distributional sense,

$\left(\square+M^{2}\right) \Delta_{F}(x)=\left(\partial_{\mu} \partial^{\mu}+M^{2}\right) \Delta_{F}(x)=-\delta^{(4)}(x)$,

and one also defines the positive- and negative-frequency Pauli-Jordan $C$-number distributions or, up to an imaginary factor, 'Wightman two-point functions',

$$
\begin{aligned}
& \Delta^{ \pm}(x)=-i\left[\varphi^{\mp}(x), \varphi^{ \pm}(0)\right]=-i\left[\varphi_{c}^{\mp}(x), \varphi_{c}^{\dagger \pm}(0)\right], \\
& \begin{aligned}
\Delta(x) & =\Delta^{+}(x)+\Delta^{-}(x)=-i[\varphi(x), \varphi(0)] \\
& =-i\left[\varphi_{c}(x), \varphi_{c}^{\dagger}(0)\right],
\end{aligned}
\end{aligned}
$$

i.e.

$\Delta^{+}(x)=-i\left\langle 0\left|\varphi^{-}(x) \varphi^{+}(0)\right| 0\right\rangle$,

$\Delta^{-}(x)=+i\left\langle 0\left|\varphi^{-}(0) \varphi^{+}(x)\right| 0\right\rangle$.

The retarded propagator is given by $\Delta^{\text {ret }}(x)=\Theta\left(x^{0}\right) \Delta(x)$, a product of distributions which is well-defined due to the harmless scaling behavior of $\Delta(x)$ at the origin $x=0$.

Some important properties of the objects and their Fourier transforms introduced so far are enlisted in the following: $\Delta(x)$ vanishes for space-like arguments $x$ with $x^{2}<0$, as required by causality. One has

$$
\begin{aligned}
& \hat{\Delta}^{ \pm}(k)=\frac{1}{(2 \pi)^{2}} \int \mathrm{d}^{4} x \Delta^{ \pm}(x) \mathrm{e}^{i k x} \\
&=\mp \frac{i}{2 \pi} \Theta\left( \pm k^{0}\right) \delta\left(k^{2}-M^{2}\right), \\
& \Delta^{+}(x)=-\Delta^{-}(-x), \\
& \Delta(x)=\Delta^{+}(x)-\Delta^{+}(-x), \\
& \Delta(-x)=-\Delta(x) .
\end{aligned}
$$




$$
\begin{aligned}
& \Delta_{F}(x)=\Theta\left(x^{0}\right) \Delta^{+}(x)-\Theta\left(-x^{0}\right) \Delta^{-}(x) . \\
& \left(\square+M^{2}\right) \Delta^{ \pm}(x)=0, \quad\left(k^{2}-M^{2}\right) \hat{\Delta}^{ \pm}(k)=0 . \\
& \Delta^{\text {ret }}=\Delta_{F}+\Delta^{-}, \\
& \Delta^{\text {ret }}(x)=\int \frac{\mathrm{d}^{4} k}{(2 \pi)^{4}} \frac{\mathrm{e}^{-i k x}}{k^{2}-M^{2}+i k^{0} 0}, \\
& \left(\square+M^{2}\right) \Delta^{\text {ret }}(x)=-\delta^{(4)}(x) .
\end{aligned}
$$

For $M=0$ the scalar Feynman propagator in configuration space is

$$
\begin{aligned}
\Delta_{F}^{0}(x) & =\int \frac{\mathrm{d}^{4} k}{(2 \pi)^{4}} \frac{\mathrm{e}^{-i k x}}{k^{2}+i 0} \\
& =\frac{i}{4 \pi^{2}} \frac{1}{x^{2}-i 0}=\frac{i}{4 \pi^{2}} P \frac{1}{x^{2}}-\frac{1}{4 \pi} \delta\left(x^{2}\right),
\end{aligned}
$$

where $P$ denotes the principal value and $\delta$ the one-dimensional Dirac distribution, and the massless Pauli-Jordan distributions in configuration space are

$$
\begin{aligned}
& \Delta^{0}(x)=-\frac{1}{2 \pi} \operatorname{sgn}\left(x^{0}\right) \delta\left(x^{2}\right), \\
& \Delta_{0}^{ \pm}(x)= \pm \frac{i}{4 \pi^{2}} \frac{1}{\left(x_{0} \mp i 0\right)^{2}-\boldsymbol{x}^{2}} .
\end{aligned}
$$

Since $\Delta^{\text {ret }}(x)=\Theta\left(x^{0}\right) \Delta(x)$, one has

$$
\Delta_{0}^{\mathrm{ret}}(x)=-\frac{1}{2 \pi} \Theta\left(x^{0}\right) \delta\left(x^{2}\right) .
$$

A notational issue concerning the principal value in the case of $\Delta_{0}^{+}$is clarified by

$$
\begin{aligned}
& \frac{1}{\left(x^{0}-i 0\right)^{2}-x^{2}} \\
& =\frac{1}{\left(\left(x^{0}-i 0\right)-|x|\right)\left(\left(x^{0}-i 0\right)+|x|\right)} \\
& =\frac{1}{2|x|} \frac{1}{x^{0}-|x|-i 0}-\frac{1}{2|x|} \frac{1}{x^{0}+|x|-i 0} \\
& =P \frac{1}{x^{2}}+i \pi \operatorname{sgn}\left(x^{0}\right) \delta\left(x^{2}\right)
\end{aligned}
$$

or

$$
\begin{aligned}
\frac{1}{\left(x^{0}-i 0\right)^{2}-x^{2}} & =\frac{1}{x^{2}-2 i 0 x^{0}-0^{2}}=\frac{1}{x^{2}-i 0 \operatorname{sgn}\left(x^{0}\right)} \\
& =P \frac{1}{x^{2}}+i \pi \operatorname{sgn}\left(x^{0}\right) \delta\left(x^{2}\right) .
\end{aligned}
$$

\subsection{Positivity}

Calculating explicitly the commutator

$$
\begin{aligned}
& {\left[\varphi_{c}(x), \varphi_{c}^{\dagger}(y)\right]} \\
& =\frac{1}{(2 \pi)^{3}} \int \frac{\mathrm{d}^{3} k^{\prime}}{\sqrt{2 E^{\prime}}} \int \frac{\mathrm{d}^{3} k}{\sqrt{2 E}} \\
& \quad \times\left[a\left(\boldsymbol{k}^{\prime}\right) \mathrm{e}^{-i k^{\prime} x}+b^{\dagger}\left(\boldsymbol{k}^{\prime}\right) \mathrm{e}^{+i k^{\prime} x}, a^{\dagger}(\boldsymbol{k}) \mathrm{e}^{+i k y}+b(\boldsymbol{k}) \mathrm{e}^{-i k y}\right]
\end{aligned}
$$

$$
\begin{aligned}
& =\frac{1}{(2 \pi)^{3}} \int \frac{\mathrm{d}^{3} k^{\prime}}{\sqrt{2 E^{\prime}}} \int \frac{\mathrm{d}^{3} k}{\sqrt{2 E}} \\
& {\left[\delta^{(3)}\left(\boldsymbol{k}^{\prime}-\boldsymbol{k}\right) \mathrm{e}^{-i k^{\prime} x+i k y}-\delta^{(3)}\left(\boldsymbol{k}^{\prime}-\boldsymbol{k}\right) \mathrm{e}^{\left.+i k^{\prime} x-i k y\right)}\right]} \\
& =\frac{1}{(2 \pi)^{3}} \int \frac{\mathrm{d}^{3} k}{2 E}\left\{\mathrm{e}^{-i k(x-y)}-\mathrm{e}^{+i k(x-y)}\right\} \\
& =\frac{1}{(2 \pi)^{3}} \int \mathrm{d}^{4} k \operatorname{sgn}\left(k^{0}\right) \delta\left(k^{2}-M^{2}\right) \mathrm{e}^{-i k(x-y)},
\end{aligned}
$$

where

$$
\begin{aligned}
\operatorname{sgn}\left(k^{0}\right) \delta\left(k^{2}-M^{2}\right) & =\operatorname{sgn}\left(k^{0}\right) \delta\left(k_{0}^{2}-\boldsymbol{k}^{2}-M^{2}\right) \\
& =\frac{\operatorname{sgn}\left(k^{0}\right)}{2\left|k^{0}\right|}\left\{\delta\left(k^{0}-E\right)+\delta\left(k^{0}+E\right)\right\}
\end{aligned}
$$

has been used, one finds one of the results given above

$$
\hat{\Delta}(k)=-\frac{i}{2 \pi} \operatorname{sgn}\left(k^{0}\right) \delta\left(k^{2}-M^{2}\right) .
$$

At the same time, at glimpse at the calculation above reveals

$$
\begin{aligned}
\hat{\Delta}^{+}(k) & =\frac{1}{(2 \pi)^{2}} \int \mathrm{d}^{4} x \Delta^{+}(x) \mathrm{e}^{i k x} \\
& =-\frac{i}{2 \pi} \Theta\left(+k^{0}\right) \delta\left(k^{2}-M^{2}\right) .
\end{aligned}
$$

Equation (58) simply expresses the fact that the scalar fields considered so far live in a Hilbert space, equipped by definition with a positive definite norm. Indeed, creating a one-particle state by acting with a smeared field operator on the vacuum

$|\Phi\rangle=\int \mathrm{d}^{4} x \Phi(x) \varphi(x)|0\rangle=\int \mathrm{d}^{4} x \Phi(x) \varphi^{+}(x)|0\rangle$

and calculating the norm gives, using Eq. (40),

$$
\begin{aligned}
\langle\Phi \mid \Phi\rangle= & i \int \mathrm{d}^{4} x^{\prime} \mathrm{d}^{4} x \Phi\left(x^{\prime}\right)^{*} \Delta^{+}\left(x^{\prime}-x\right) \Phi(x) \\
= & \frac{i}{(2 \pi)^{6}} \int \mathrm{d}^{4} k^{\prime \prime} \mathrm{d}^{4} k^{\prime} \mathrm{d}^{4} k \mathrm{~d}^{4} x^{\prime} \mathrm{d}^{4} x \\
& \times \hat{\Phi}\left(-k^{\prime}\right)^{*} \mathrm{e}^{-i k^{\prime} x^{\prime}} \hat{\Delta}^{+}\left(k^{\prime \prime}\right) \mathrm{e}^{-i k^{\prime \prime}\left(x^{\prime}-x\right)} \hat{\Phi}(k) \mathrm{e}^{-i k x},
\end{aligned}
$$

where the non-vanishing test function and the positivefrequency Pauli-Jordan distribution have been replaced their corresponding Fourier transforms. Using the distributional identity

$$
\int \mathrm{d}^{4} k \mathrm{e}^{+i k x}=(2 \pi)^{4} \delta^{(4)}(x)
$$

is allowed here and leads to

$$
\begin{aligned}
\langle\Phi \mid \Phi\rangle= & i(2 \pi)^{2} \int \mathrm{d}^{4} k^{\prime \prime} \mathrm{d}^{4} k^{\prime} \mathrm{d}^{4} k \\
& \times \hat{\Phi}\left(-k^{\prime}\right)^{*} \hat{\Delta}^{+}\left(k^{\prime \prime}\right) \hat{\Phi}(k) \delta^{(4)}\left(k^{\prime}+k^{\prime \prime}\right) \delta^{(4)}\left(k-k^{\prime \prime}\right)
\end{aligned}
$$




$$
\begin{aligned}
& =i(2 \pi)^{2} \int \mathrm{d}^{4} k^{\prime \prime} \hat{\Phi}\left(k^{\prime \prime}\right)^{*} \hat{\Delta}^{+}\left(k^{\prime \prime}\right) \hat{\Phi}\left(k^{\prime \prime}\right) \\
& =2 \pi \int \mathrm{d}^{4} k \Theta\left(+k^{0}\right) \delta\left(k^{2}-M^{2}\right) \hat{\Phi}(k)^{*} \hat{\Phi}(k)>0,
\end{aligned}
$$

i.e., the Heaviside- and $\delta$-distributions in Eq. (59) express the fact that states created by bosonic scalar field operators have positive norm.

We will see below that the derivative coupling model can also be quantized by using fermionic scalar fields, i.e. ghosts, which exhibit some properties invoking some conceptual differences to the discussion above.

\section{The derivative coupling model: bosonic version}

\subsection{General considerations}

The transition from the classical derivative coupling model according to Eqs. (3) and (4) to a quantized version generates a problem. The exponential

$\mathrm{e}^{-i g \varphi(x)}=\sum_{n=0}^{\infty} \frac{(-i g \varphi(x))^{n}}{n !}$

is not well-defined as an operator-valued distribution, since already $\varphi(x) \varphi(x)$ is ill-defined. For example, a short calculation shows that $\langle 0|\varphi(x) \varphi(x)| 0\rangle$ is a divergent expression which has to be regularized. A way out of this situation is offered by the normal ordering of field operators which corresponds to a recursive point-splitting regularization

$: \varphi(x):=\varphi(x)$

$: \varphi(x)^{2}:=\lim _{y \rightarrow x}[\varphi(x) \varphi(y)-\langle 0|\varphi(x) \varphi(y)| 0\rangle]$,

$: \varphi(x)^{n}:=\lim _{y \rightarrow x}\left[: \varphi(x)^{n-1}: \varphi(y)\right.$

$$
\left.-(n-1)\langle 0|\varphi(x) \varphi(y)| 0\rangle: \varphi(x)^{n-2}:\right] .
$$

The normally ordered product : $\varphi(x)^{n}:$ is an operatorvalued distributions, as well as the tensor product : $\varphi(x)^{n}$ : $: \varphi(y)^{n}:[7]$.

Literally, normal ordering products of free field operators moves all destruction operators to the right, so that creation operators are moved to the left. For example,

$$
\begin{aligned}
& \varphi(x) \varphi(y) \\
&=\left(\varphi^{-}(x)+\varphi^{+}(x)\right)\left(\varphi^{-}(y)+\varphi^{+}(y)\right) \\
&= \varphi^{-}(x) \varphi^{-}(y)+\varphi^{+}(x) \varphi^{+}(y)+\varphi^{+}(x) \varphi^{-}(y) \\
&+\varphi^{+}(y) \varphi^{-}(x)+\left[\varphi^{-}(x), \varphi^{+}(y)\right] \\
&=: \varphi(x) \varphi(y):+i \Delta^{+}(x-y) .
\end{aligned}
$$

Calculating the following vacuum expectation value according to Wick's theorem $\left\langle 0\left|: \varphi(x)^{n}:: \varphi(0)^{n}:\right| 0\right\rangle=i^{n} n ! \Delta^{+}(x)^{n}$,

is a well-defined procedure, and the expressions

$\frac{(-i g)^{n}: \varphi(x)^{n}:}{n !}$

are well-defined composite field operators. But still, the sum

$: \mathrm{e}^{-i g \varphi(x)}:=\sum_{n=0}^{\infty} \frac{(-i g)^{n}: \varphi(x)^{n}:}{n !}=\mathrm{e}^{-i g \varphi^{+}(x)} \mathrm{e}^{-i g \varphi^{-}(x)}$

turns out to be 'harmless' only in $1+1$ dimensions. For the sake of completeness, some basic facts concerning the derivative coupling model in two space-time dimensions as discussed by Schroer [4] are provided in the following.

4.2 The derivative coupling model in two dimensions

In $1+1$ dimensions, the neutral scalar field

$$
\begin{aligned}
\varphi(x) & =\varphi^{-}(x)+\varphi^{+}(x) \\
& =\frac{1}{\sqrt{2 \pi}} \int \frac{\mathrm{d} k^{1}}{\sqrt{2 k^{0}}}\left[a(\boldsymbol{k}) \mathrm{e}^{-i k x}+a^{\dagger}(\boldsymbol{k}) \mathrm{e}^{+i k x}\right]
\end{aligned}
$$

leads to the two-dimensional positive frequency PauliJordan distribution

$$
\begin{aligned}
\Delta^{+}(x-y) & =-i\langle 0|\varphi(x) \varphi(y)| 0\rangle \\
& =-\frac{i}{2 \pi} \int \mathrm{d}^{2} k \Theta\left(k^{0}\right) \delta\left(k^{2}-M^{2}\right) \mathrm{e}^{-i k(x-y)} \\
& =-\frac{i}{2 \pi} \int \frac{\mathrm{d} k^{1}}{2 k^{0}} \mathrm{e}^{-i k(x-y)} \\
& =-\frac{i}{2 \pi} K_{0}\left(M \sqrt{-(x-y)^{2}+i\left(x^{0}-y^{0}\right) 0}\right) .
\end{aligned}
$$

This integral diverges for $M \rightarrow 0$, since the modified Bessel function (or MacDonald function) behaves for $0<$ $x \ll 1$ like

$K_{0}(x) \simeq-\ln \left(\frac{x}{2}\right)-\gamma$

where $\gamma$ denotes the Euler-Mascheroni constant. Regularizing in the infrared according to

$\Delta^{+}(x ; \lambda)=-\frac{i}{2 \pi} \int \frac{\mathrm{d} k^{1}}{2\left|k^{1}\right|} \Theta\left(\left|k^{1}\right|-\lambda\right) \mathrm{e}^{-i k x}$ 
leads to $(0<\lambda \ll 1)$

$$
\begin{aligned}
\Delta^{+}(x ; \lambda) & \simeq \frac{i}{4 \pi} \ln \left(-\mu^{2} x^{2}+i x^{0} 0\right) \\
& =\frac{i}{4 \pi} \ln \left(-x^{2}+i x^{0} 0\right)+C
\end{aligned}
$$

with $\mu=e^{\gamma} \lambda$.

On the restricted space of test functions

$\mathcal{K}=\left\{f(x) \in \mathcal{S}\left(\mathbb{R}^{2}\right) \mid \int \mathrm{d}^{2} x f(x)=0\right\}$

the massless field $\varphi(x)$ is an operator-valued distribution, as well as

$\Delta_{\text {reg }}^{+}(x)=\frac{i}{4 \pi} \ln \left(-x^{2}+i x^{0} 0\right)$.

Therefore, one has

$$
\begin{aligned}
\langle 0| & : \mathrm{e}^{-i g \varphi(x)}:: \mathrm{e}^{+i g \varphi(y)}:|0\rangle \\
& =\sum_{n=0}^{\infty} \frac{i^{n}}{n !}\left(g^{2}\right)^{n}\left[\Delta_{\text {reg }}^{+}(x-y)\right]^{n}=\mathrm{e}^{i g^{2} \Delta_{\text {reg }}^{+}(x-y)}
\end{aligned}
$$

and

$$
\begin{aligned}
& \langle 0|\psi(x) \bar{\psi}(y)| 0\rangle \\
& =\left\langle 0\left|\psi_{0}(x) \bar{\psi}_{0}(y)\right| 0\right\rangle\left\langle 0\left|: \mathrm{e}^{-i g \varphi(x)}:: \mathrm{e}^{+i g \varphi(y)}:\right| 0\right\rangle \\
& =\left\langle 0\left|\psi_{0}(x) \bar{\psi}_{0}(y)\right| 0\right\rangle \mathrm{e}^{-\frac{g^{2}}{4 \pi} \ln \left(-(x-y)^{2}+i\left(x^{0}-y^{0}\right) 0\right)} \\
& =\left\langle 0\left|\psi_{0}(x) \bar{\psi}_{0}(y)\right| 0\right\rangle\left(\frac{1}{-(x-y)^{2}+i\left(x^{0}-y^{0}\right) 0}\right)^{g^{2} / 4 \pi}
\end{aligned}
$$

where $\psi_{0}$ denotes the free fermionic field in two space-time dimensions. A straightforward calculation [4] also shows that

$\Delta_{F}^{\psi}(k)=\langle 0| T\left(\psi(x) \bar{\psi}(y)|0\rangle \sim\left(k^{2}-m^{2}\right)^{g^{2} / 2 \pi-1}\right.$.

No meromorphic pole structure appears for $g \neq 0$, although the $S$-matrix of the theory is trivial. For this reason, Schroer coined the expression infraparticle for the states described by the dressed field $\psi(x)$.

\subsection{Four-dimensional aspects}

In $3+1$ dimensions one has $\Delta_{0}^{+}(x)=\frac{i}{4 \pi^{2}} \frac{1}{\left(x_{0}-i 0\right)^{2}-x^{2}}$, and

$$
\begin{aligned}
D^{+}(x-y) & =\left\langle 0\left|: \mathrm{e}^{-i g \varphi(x)}:: \mathrm{e}^{+i g \varphi(y)}:\right| 0\right\rangle \\
& =\sum_{n=0}^{\infty} \frac{i^{n}}{n !}\left(g^{2}\right)^{n}\left[\Delta_{0}^{+}(x-y)\right]^{n} \\
& =\mathrm{e}^{i g^{2} \Delta_{0}^{+}(x-y)}=\exp \left(-\frac{g^{2}}{4 \pi^{2}\left(\left(x_{0}-i 0\right)^{2}-x^{2}\right)}\right)
\end{aligned}
$$

is a highly ultraviolet-divergent (still formal) expression as can be anticipated from the singular behavior in configuration space for $x \rightarrow 0$. In fact, the exponential of a free scalar field operator in four space-time dimensions is no longer an operator-valued distribution defined on $\mathcal{S}\left(\mathbb{R}^{4}\right)$.

In conventional regularization theory, one would regularize the exponential of a scalar field according to

$$
\begin{aligned}
& \mathrm{e}^{-i g \varphi(x)} \rightarrow \mathrm{e}^{-\frac{i}{2} g^{2} \Delta_{\Lambda}^{+}(0)} \mathrm{e}^{-i g \varphi_{\Lambda}(x)} \stackrel{\Lambda \rightarrow \infty}{\rightarrow}: \mathrm{e}^{-i g \varphi(x)}: \\
& =\lim _{\Lambda \rightarrow 0}: \mathrm{e}^{-i g \varphi_{\Lambda}(x)}:=\mathrm{e}^{-i g \varphi_{\Lambda}^{+}(x)} \mathrm{e}^{-i g \varphi_{\Lambda}^{-}(x)},
\end{aligned}
$$

with a scalar field $\varphi_{\Lambda}(x)$ with ultraviolet cutoff $\Lambda$ generating a regular two-point distribution $\Delta_{\Lambda}^{+}(x)$. The field $\psi_{u n, \Lambda}(x)=$ $\psi_{0} \mathrm{e}^{-i g \varphi_{\Lambda}(x)}$ would not converge to a well-defined operatorvalued distribution in any sense. However, one can write for the renormalized field with ultraviolet cutoff

$$
\begin{aligned}
\psi_{\text {ren, }, \Lambda}(x) & =: \mathrm{e}^{-i g \varphi_{\Lambda}(x)}: \psi_{0}(x)=\mathrm{e}^{-\frac{i}{2} g^{2} \Delta_{\Lambda}^{+}(0)} \psi_{u n, \Lambda}(x) \\
& =Z_{\Lambda}^{-1 / 2} \psi_{u n, \Lambda}(x) .
\end{aligned}
$$

In the limit $\Lambda \rightarrow \infty$, with $\psi_{\text {un }}$ as the unrenormalized formal limit of $\psi_{\Lambda}$, one has formally

$\psi_{\text {ren }}(x)=\lim _{\Lambda \rightarrow \infty} Z_{\Lambda}^{-1 / 2} \psi_{u n, \Lambda}=Z^{-1 / 2} \psi_{\text {un }}(x)$,

where

$Z_{\Lambda}^{-1 / 2}=\mathrm{e}^{-\frac{i}{2} g^{2} \Delta_{\Lambda}^{+}(0)}$.

Then

$\left\{\psi_{\text {ren }, \alpha}\left(x^{0}, \boldsymbol{x}\right), \bar{\psi}_{\text {ren }, \beta}\left(x^{0}, \boldsymbol{y}\right)\right\}=Z^{-1}\left(\gamma^{0}\right)_{\alpha \beta} \delta^{(3)}(\boldsymbol{x}-\boldsymbol{y})$,

i.e. the standard equal time anti-commutation relations cannot be fulfilled by the renormalized fields since $Z \rightarrow \infty$, but the renormalized field $\psi_{\text {ren }}$ has well-defined correlation functions. The distribution $\mathrm{e}^{i g^{2} \Delta^{+}(x-y)}$ cannot be restricted to equal times, $x^{0}=y^{0}$, a non-canonical property which one expects for interacting fields.

Still, perturbative terms like $\Delta_{0}^{+}(x)^{n}$ can be defined without problems. In the following, the product in configuration space $\Delta_{0}^{+}(x)^{2}$ is investigated in detail in configuration as well as in momentum space. Defining $\Delta_{2}^{+}(x)=\left(\Delta_{0}^{+}(x)\right)^{2}$, one calculates

$$
\begin{aligned}
\mathcal{F} & \left(\Delta_{0}^{+}(x)^{2}\right)(k) \\
= & \frac{1}{(2 \pi)^{2}} \int \mathrm{d}^{4} x \mathrm{e}^{+i k x} \frac{1}{(2 \pi)^{2}} \int \mathrm{d}^{4} k^{\prime} \\
& \times \hat{\Delta}_{0}^{+}\left(k^{\prime}\right) \mathrm{e}^{-i k^{\prime} x} \frac{1}{(2 \pi)^{2}} \int \mathrm{d}^{4} k^{\prime \prime} \hat{\Delta}_{0}^{+}\left(k^{\prime \prime}\right) \mathrm{e}^{-i k^{\prime \prime} x},
\end{aligned}
$$


and using

$$
\int \mathrm{d}^{4} x \mathrm{e}^{i\left(k-k^{\prime}-k^{\prime \prime}\right) x}=(2 \pi)^{4} \delta^{(4)}\left(k-k^{\prime}-k^{\prime \prime}\right)
$$

this implies

$$
\begin{aligned}
& \hat{\Delta}_{2}^{+}(k) \\
& =\frac{1}{(2 \pi)^{2}} \int \mathrm{d}^{4} k^{\prime} \mathrm{d}^{4} k^{\prime \prime} \hat{\Delta}_{0}^{+}\left(k^{\prime}\right) \hat{\Delta}_{0}^{+}\left(k^{\prime \prime}\right) \delta^{(4)}\left(k-k^{\prime}-k^{\prime \prime}\right) \\
& =\frac{1}{(2 \pi)^{2}} \int \mathrm{d}^{4} k^{\prime} \hat{\Delta}_{0}^{+}\left(k^{\prime}\right) \hat{\Delta}_{0}^{+}\left(k-k^{\prime}\right) \\
& =-(2 \pi)^{-4} \int \mathrm{d}^{4} k^{\prime} \Theta\left(k^{\prime 0}\right) \delta\left(k^{\prime 2}\right) \Theta\left(k^{0}-k^{\prime 0}\right) \delta\left(\left(k-k^{\prime}\right)^{2}\right) .
\end{aligned}
$$

The integral Eq. (90) vanishes if $k^{0} \notin \bar{V}^{+}$, i.e. if $k$ is not in the closed forward light-cone

$$
\bar{V}^{+}=\left\{k \mid k^{0} \geq 0, k^{2} \geq 0\right\} .
$$

In a Lorentz system where $k=\left(k^{0}>0, \mathbf{0}\right)$, due to the first $\Theta$ - and $\delta$-distribution in Eq. (90) one has $E=\left|\boldsymbol{k}^{\prime}\right|=k_{0}^{\prime}$ and

$$
\delta\left(\left(k-k^{\prime}\right)^{2}\right)=\delta\left(\left(k_{0}-k_{0}^{\prime}\right)^{2}-E^{2}\right)=\delta\left(k_{0}^{2}-2 k_{0} E\right),
$$

and therefore

$$
\begin{aligned}
\hat{\Delta}_{2}^{+}(k) & =-(2 \pi)^{-4} \int \frac{\mathrm{d}^{3} k^{\prime}}{2 E} \Theta\left(k^{0}-E\right) \delta\left(k_{0}^{2}-2 k^{0} E\right) \\
& =-(2 \pi)^{-4} \int d\left|\boldsymbol{k}^{\prime}\right| \frac{4 \pi\left|\boldsymbol{k}^{\prime}\right|^{2}}{2\left|\boldsymbol{k}^{\prime}\right|} \Theta\left(k^{0}-\left|\boldsymbol{k}^{\prime}\right|\right) \frac{\delta\left(k^{0} / 2-\left|\boldsymbol{k}^{\prime}\right|\right)}{\left|2 k^{0}\right|} \\
\left|\boldsymbol{k}^{\prime}\right| & \stackrel{=k^{0} / 2}{=}-\frac{1}{4(2 \pi)^{3}} \Theta\left(k^{0}\right) .
\end{aligned}
$$

For arbitrary $k$ it follows that

$$
\hat{\Delta}_{2}^{+}(k)=-\frac{1}{4(2 \pi)^{3}} \Theta\left(k^{0}\right) \Theta\left(k^{2}\right)
$$

As a further step, the meaning of the expression $\Delta_{n}^{+}(x)=$ $\left(\Delta_{0}^{+}(x)\right)^{n}$ is investigated in configuration space. Obviously, $\Delta_{n}^{+}(x)=\left(\Delta_{0}^{+}(x)\right)^{n} \sim 1 / x^{2 n}=1 /\left(x^{2}\right)^{n}$ is very 'singular' in $\mathrm{x}$-space. For $n \geq 2$ and $x^{2} \neq 0$ one easily derives

$$
\square \frac{1}{\left(\left(x^{0}-i \varepsilon\right)^{2}-x^{2}\right)^{n}}=\frac{4 n(n-1)}{\left(\left(x^{0}-i \varepsilon\right)^{2}-x^{2}\right)^{n+1}}
$$

translating into

$$
\square \Delta_{n}^{+}(x)=-16 i \pi^{2} n(n-1) \Delta_{n+1}^{+}(x) .
$$

In momentum space, this implies

$$
-k^{2} \hat{\Delta}_{n}^{+}(k)=-16 i \pi^{2} n(n-1) \hat{\Delta}_{n+1}^{+}(k)
$$

or

$\hat{\Delta}_{n+1}^{+}(k)=\frac{k^{2}}{16 i \pi^{2} n(n-1)} \hat{\Delta}_{n}^{+}(k)$

and inductively it follows for $n \geq 2$ that

$\hat{\Delta}_{n}^{+}(k)=\frac{(-i)^{n}\left(k^{2}\right)^{n-2}}{4^{n-1}(2 \pi)^{2 n-1}(n-1) !(n-2) !} \Theta\left(k^{0}\right) \Theta\left(k^{2}\right)$.

Hence, the Fourier transform of

$$
\begin{aligned}
D^{+}(x-y) & =\left\langle 0\left|: \mathrm{e}^{-i g \varphi(x)}:: \mathrm{e}^{+i g \varphi(y)}:\right| 0\right\rangle \\
& =\sum_{n=0}^{\infty} \frac{i^{n}}{n !}\left(g^{2}\right)^{n}\left[\Delta_{0}^{+}(x-y)\right]^{n}
\end{aligned}
$$

sums up to

$$
\begin{aligned}
\hat{D}^{+}(k)= & (2 \pi)^{2} \delta^{(4)}(k)+\frac{g^{2}}{2 \pi} \Theta\left(k^{0}\right) \delta\left(k^{2}\right) \\
& +\frac{g^{4}}{2 ! 4(2 \pi)^{3}} \Theta\left(k^{0}\right) \Theta\left(k^{2}\right)+\cdots \\
= & (2 \pi)^{2} \delta^{(4)}(k)+\frac{g^{2}}{2 \pi} \Theta\left(k^{0}\right) \delta\left(k^{2}\right) \\
& +\sum_{n=2}^{\infty} \frac{2\left(g^{2}\right)^{n}\left(k^{2}\right)^{n-2}}{(4 \pi)^{2 n-1} n !(n-1) !(n-2) !} \Theta\left(k^{0}\right) \Theta\left(k^{2}\right) .
\end{aligned}
$$

This expression is, up to a normalization constant, the correct expression for Eq. (14) in [8], where the combinatorial coefficients are stated incorrectly without a derivation.

In order to highlight the high-energy behavior of the above expression we introduce the function

$\mathrm{d}(x)=\sum_{n=2}^{\infty} \frac{x^{n}}{n !(n-1) !(n-2) !}$

For $x \gg 1, d(x)$ asymptotically behaves like

$\mathrm{d}(x) \sim \frac{1}{2 \pi \sqrt{3}} x^{2 / 3} \mathrm{e}^{3 x^{1 / 3}}$

The derivation of this result is given in Appendix B. $\hat{D}^{+}(k)$ grows faster than any polynomial on the momentum-space forward light-cone. Therefore, $\hat{D}^{+}$does not belong to the Schwartz space of tempered distributions, since an integral of the form

$\hat{D}^{+}(\hat{g})=\int \mathrm{d}^{4} k \hat{D}^{+}(k) \hat{g}(k)$ 
does not exist for all $g, \hat{g} \in \mathcal{S}\left(\mathbb{R}^{4}\right)$, despite the rapid decrease of such functions. However, the integral Eq. (104) exists if $\hat{g}$ is of compact support. Unfortunately, a non-vanishing Fourier transform $\hat{g}(k)$ implies that $g(x)$ does not have a compact support in configuration space, which hampers the definition of causality according to Eq. (33).

However, Jaffe [9] has shown that it is still possible to construct a restricted space of test functions in configuration space which contains test functions of compact support, such that the principle of causality can be formulated and the fields in the derivative coupling model can be considered operator-valued distributions on the appropriately chosen test function space; it is possible to find test functions with compact support which have a Fourier transform decreasing so fast that integral like the one in Eq. (104) exist. One finally may conclude that even a physically trivial interaction may enforce a formalism which goes beyond the well-behaved setting of Schwartz distributions, which lies at the basis of perturbatively renormalizable QFTs.

\subsection{Operator field equations of motion}

Equation (3) contains the product of two field operators. A 'subtraction' or regularization is necessary to define the equations of motion of the derivative coupling model. In fact, normal ordering in the sense of a subtraction leads to

$$
\begin{aligned}
& \left(\partial_{\mu} \varphi \gamma^{\mu} \psi\right)_{\text {reg }}(x) \\
& =: \partial_{\mu} \varphi(x) \gamma^{\mu} \psi(x): \\
& =\lim _{y \rightarrow x}\left[\partial_{\mu} \varphi(x) \gamma^{\mu} \psi(y)-\left\langle 0\left|\partial_{\mu} \varphi(x) \gamma^{\mu} \psi(y)\right| 0\right\rangle\right] \\
& =\lim _{y \rightarrow x}\left[: \partial_{\mu} \varphi(x):: \gamma^{\mu} \psi_{0}(x) \mathrm{e}^{-i g \varphi(x)}:\right. \\
& \left.\quad-g \partial_{\mu}^{x} \Delta^{+}(x-y) \gamma^{\mu}: \psi_{0}(x) \mathrm{e}^{-i g \varphi(x)}:\right] .
\end{aligned}
$$

\section{5 'Fermionic' version of the derivative coupling model}

\subsection{Gauge charge operator for free fields}

Before turning back to the derivative coupling model, some remarks concerning the gauge structure of perturbative quantum electrodynamics (QED) are in order. Considering the free massless neutral vector potential prominent in QED obeying the wave equation $\square A^{\mu}(x)=0$ in Feynman gauge, the Fourier representation reads $\left(\omega=k^{0}=|\boldsymbol{k}|=\right.$ $\sqrt{k_{1}^{2}+k_{2}^{2}+k_{3}^{2}}$

$$
A^{\mu}(x)=(2 \pi)^{-3 / 2} \int \frac{\mathrm{d}^{3} k}{\sqrt{2 \omega}}\left(a^{\mu}(\boldsymbol{k}) \mathrm{e}^{-i k x}+a^{\mu}(\boldsymbol{k})^{\dagger} \mathrm{e}^{i k x}\right),
$$

and can be quantized in Lorentz-invariant form according to

$\left[A^{\mu}(x), A^{v}(y)\right]=-i g^{\mu \nu} \Delta_{0}(x-y)$.

The commutators of the absorption and emission parts alone are

$\left[A_{-}^{\mu}(x), A_{+}^{v}(y)\right]=-i g^{\mu \nu} \Delta_{0}^{+}(x-y)$,

$\left[A_{+}^{\mu}(x), A_{-}^{v}(y)\right]=-i g^{\mu \nu} \Delta_{0}^{-}(x-y)$.

In classical electrodynamics the vector potential can be changed by a gauge transformation

$A^{\prime \mu}(x)=A^{\mu}(x)+\lambda \partial^{\mu} u(x)$,

with $u(x)$ again fulfilling the wave equation $\square u(x)=0$ since the transformed field $A^{\prime \mu}(x)$ still should satisfy the original wave equation and the same commutation relations Eq. (107) as $A^{\mu}(x)$. This is true if the gauge transformation Eq. (110) is of the following form:

$A^{\prime \mu}(x)=\mathrm{e}^{-i \lambda Q} A^{\mu}(x) \mathrm{e}^{i \lambda Q}$,

where $Q$ is some operator in the Fock-Hilbert space the photon field lives in. Expanding Eq. (111) by means of the Lie series

$=A^{\mu}(x)-i \lambda\left[Q, A^{\mu}(x)\right]+O\left(\lambda^{2}\right)$

and a comparison with Eq. (110) leads to the condition

$\left[Q, A^{\mu}(x)\right]=i \partial^{\mu} u(x)$.

The operator $Q$ will be called gauge charge because it is the infinitesimal generator of the gauge transformation defined by Eq. (110). Its importance relies on the fact that the factor space given by the kernel and the closure of the range of the gauge operator $\mathcal{F}_{\mathrm{ph}}=\operatorname{Ker} Q / \overline{\operatorname{Ran} \mathrm{Q}}$ is isomorphic to the subspace of physical photon states $[10,11]$. Before clarifying what this means, the following remarks are in order.

Firstly, it is not clear at the present status of the discussion whether the field introduced in Eq. (110) has to be considered as a classical C-number field or a quantum field. It will turn out that it can be treated as a classical or a quantized bosonic field in QED, however, for non-abelian gauge theories like quantum chromodynamics (QCD) the $u$-field necessarily becomes a fermionic scalar field, also called a ghost field. We will call the massless scalar field $u$ a ghost field in the following, irrespective of the fact whether it is quantized or not, bosonic or fermionic.

Secondly, the commutator given in Eq. (107) generates a problem for $\mu=v=0: g^{00}$ has the wrong sign, making it impossible to have time-like photon states with positive norm 
if one insists on the hermiticity of the $A^{0}$-field component. The positive frequency Pauli-Jordan distribution for timelike photons would acquire the opposite sign as exhibited by Eq. (59). The situation is remedied by defining a so-called Krein structure $[11,12]$ on the photonic Fock-Hilbert space. Introducing a conjugation $K$

$a_{0}(\boldsymbol{k})^{K}=-a_{0}(\boldsymbol{k})^{\dagger}, \quad a_{j}(\boldsymbol{k})^{K}=a_{j}(\boldsymbol{k})^{\dagger}, \quad j=1,2,3$,

so that $A_{\mu}^{K}=A_{\mu}$, allows one to maintain the positivedefiniteness on the Fock-Hilbert space which is comprised in the definition of a Hilbert space, however, the redefined field

$A^{0}(x)=(2 \pi)^{-3 / 2} \int \frac{\mathrm{d}^{3} k}{\sqrt{2 \omega}}\left(a^{0}(\boldsymbol{k}) \mathrm{e}^{-i k x}-a^{0}(\boldsymbol{k})^{\dagger} \mathrm{e}^{i k x}\right)=A_{K}^{0} s$

which will be used from now on is no longer a hermitian field. In accordance with the commutation relations Eq. (107) holds

$\left[a^{\mu}(\boldsymbol{k}), a^{v}\left(\boldsymbol{k}^{\prime}\right)^{\dagger}\right]=\delta^{\mu v} \delta^{(3)}\left(\boldsymbol{k}-\boldsymbol{k}^{\prime}\right)$,

$\left[a^{\mu}(\boldsymbol{k}), a^{\nu}\left(\boldsymbol{k}^{\prime}\right)\right]=\left[a^{\mu}(\boldsymbol{k})^{\dagger}, a^{\nu}\left(\boldsymbol{k}^{\prime}\right)^{\dagger}\right]=0$.

Fortunately, abandoning the hermiticity of the zeroth component of the gauge potential does not invalidate the unitarity of the $S$-matrix in QED on the physical subspace of transverse photons [10].

The gauge transformation operator with the properties required so far turns out to be

$Q=\int_{x_{0}=\text { const. }} \mathrm{d}^{3} x \partial_{\mu} A^{\mu}(x) \stackrel{\leftrightarrow}{\partial_{0}} u(x)$.

$Q$ has the physical dimension of a scalar or vector field, an energy, or an inverse length squared. It is sufficient for the moment to consider $u$ as a real C-number field. In any case anticipated so far it can be shown that $\mathrm{Q}$ is a well-defined operator on the Fock space. It is not important over which space-like plane the integral in Eq. (118) is taken, since $Q$ is time independent:

$$
\begin{aligned}
\dot{Q} & =\int_{x_{0}=\text { const. }} \mathrm{d}^{3} x\left(-\partial_{0}^{2} \partial_{\mu} A^{\mu} u+\partial_{\mu} A^{\mu} \partial_{0}^{2} u\right) \\
& =\int_{x_{0}=\text { const. }} \mathrm{d}^{3} x\left(-\triangle \partial_{\mu} A^{\mu} u+\partial_{\mu} A^{\mu} \triangle u\right)=0 .
\end{aligned}
$$

This formal proof uses the wave equation and partial integration. Another way to understand the time independence of the gauge charge is to define the gauge current $j_{g}^{\mu}=\partial_{\nu} A^{\nu} \stackrel{\leftrightarrow}{\partial^{\mu}} u, \quad Q=\int \mathrm{d}^{3} x j_{g}^{0}$,

which is conserved

$\partial_{\mu} j_{g}^{\mu}=\partial_{\mu}\left(\partial_{\nu} A^{\nu} \partial^{\mu} u-\partial^{\mu} \partial_{\nu} A^{v} u\right)=0$.

Besides the crucial property of the gauge charge expressed by the commutator with $A^{\mu}$

$\left[Q, A^{\mu}(x)\right]=i \partial^{\mu} u(x)$,

all higher commutators like

$\left[Q,\left[Q, A^{\mu}(x)\right]\right]=0$

vanish for a bosonic or C-number ghost field $u$, but not for a fermionic ghost field. Equation (122) can be derived by using some distributional properties of the massless Pauli-Jordan distribution

$\Delta^{0}(x)=-\frac{i}{(2 \pi)^{3}} \int \mathrm{d}^{4} k \delta\left(k^{2}\right) \operatorname{sgn}\left(k_{0}\right) \mathrm{e}^{-i k x}$.

Using the identity

$\delta\left(k^{2}\right)=\delta\left(k_{0}^{2}-\boldsymbol{k}^{2}\right)=\frac{1}{2\left|k^{0}\right|}\left(\delta\left(k^{0}-|\boldsymbol{k}|\right)+\delta\left(k^{0}+|\boldsymbol{k}|\right)\right)$,

leads to

$$
\begin{aligned}
\partial_{0} \Delta^{0}(x)= & -\frac{i}{(2 \pi)^{3}} \int \frac{\mathrm{d}^{4} k}{2\left|k^{0}\right|} \\
& \times\left(\delta\left(k^{0}-|\boldsymbol{k}|\right)-\delta\left(k^{0}+|\boldsymbol{k}|\right)\left(-i k^{0}\right) \mathrm{e}^{-i k x}\right. \\
= & -\frac{1}{2(2 \pi)^{3}} \int \mathrm{d}^{3} k\left(\mathrm{e}^{-i\left(|\boldsymbol{k}| x^{0}-\boldsymbol{k x}\right)}+\mathrm{e}^{-i\left(-|\boldsymbol{k}| x^{0}-\boldsymbol{k} \boldsymbol{x}\right)}\right) .
\end{aligned}
$$

Restricting this result to $x^{0}=0$ implies

$\left.\partial_{0} \Delta^{0}(x)\right|_{x^{0}=0}=-(2 \pi)^{-3} \int \mathrm{d}^{3} k \mathrm{e}^{+i k \boldsymbol{x}}=-\delta^{(3)}(\boldsymbol{x})$.

In a completely analogous way, one derives for the derivatives of the Pauli-Jordan distribution restricted to the space-like plane $x^{0}=0$

$\left.\partial_{0}^{2} \Delta^{0}(x)\right|_{x^{0}=0}=0,\left.\quad \nabla \Delta^{0}(x)\right|_{x^{0}=0}=0$.

Note that we always consider the well-defined differentiated distribution first, which then gets restricted to a subset of its support. The commutator is now given explicitly by 


$$
\begin{aligned}
{\left[Q, A_{\mu}(y)\right] } & =\left[\int_{x^{0}=y^{0}} \mathrm{~d}^{3} x \partial_{\nu} A^{v}(x) \stackrel{\leftrightarrow}{\partial_{0}^{x}} u(x), A_{\mu}(y)\right] \\
& =-i \int_{x^{0}=y^{0}} \mathrm{~d}^{3} x \partial_{\mu}^{x} \Delta^{0}(x-y) \stackrel{\leftrightarrow}{\partial_{0}^{x}} u(x) .
\end{aligned}
$$

Here, use was made of the freedom to choose any constant value for $x^{0}$. Setting $x^{0}=y^{0}$, such that $x^{0}-y^{0}=0$ and applying Eqs. (127) and (128) in the sequel, one has for $\mu=0$

$$
\begin{aligned}
{\left[Q, A_{0}(y)\right] } & =-i \int_{x^{0}=y^{0}} \mathrm{~d}^{3} x \partial_{0}^{x} \Delta^{0}(x-y) \overleftrightarrow{\partial_{0}^{x}} u(x) \\
& =i \int_{x^{0}=y^{0}} \mathrm{~d}^{3} x \delta^{(3)}(\boldsymbol{x}-\boldsymbol{y}) \partial_{0}^{x} u(x)=i \partial_{0} u(y)
\end{aligned}
$$

due to the double time-like derivative of $\Delta^{0}$ vanishing on the integration domain according to Eq. (128). The result for the commutator of $Q$ with the space-like components of $A^{\mu}$ is also obtained by using Eqs. (127) and (128) and by shifting the gradient acting of the Pauli-Jordan distribution by partial integration on the ghost field.

From the Lie series

$$
\begin{aligned}
& \mathrm{e}^{-i \lambda Q} A^{\mu} \mathrm{e}^{+i \lambda Q} \\
& \quad=A^{\mu}-\frac{i \lambda}{1 !}\left[Q, A^{\mu}\right]-\frac{\lambda^{2}}{2 !}\left[Q,\left[Q, A^{\mu}\right]\right]+\cdots \\
& =A^{\mu}-i \lambda\left[Q, A^{\mu}\right]=A^{\mu}+\lambda \partial^{\mu} u,
\end{aligned}
$$

it follows that $Q$ is indeed a generator of gauge transformations for a $\mathrm{C}$-number ghost field $u$; it is a simple task to show that also $\left[Q, i \partial^{\mu} u\right]=\left[Q,\left[Q, A^{\mu}\right]\right]=0$ holds in the case of a bosonic massless ghost field.

As a further step fermionic ghost fields are introduced. $u(x)$ is assumed to be a fermionic scalar field with mass zero which has the following Fourier decomposition $(\omega(\boldsymbol{k})=$ $|k|)$ :

$u(x)=(2 \pi)^{-3 / 2} \int \frac{\mathrm{d}^{3} k}{\sqrt{2 \omega(\boldsymbol{k})}}\left(c_{2}(\boldsymbol{k}) \mathrm{e}^{-i k x}+c_{1}^{\dagger}(\boldsymbol{k}) \mathrm{e}^{i k x}\right)$,

and in addition, a further scalar field shall be defined by

$\tilde{u}(x)=(2 \pi)^{-3 / 2} \int \frac{\mathrm{d}^{3} k}{\sqrt{2 \omega(\boldsymbol{k})}}\left(-c_{1}(\boldsymbol{k}) \mathrm{e}^{-i k x}+c_{2}^{\dagger}(\boldsymbol{k}) \mathrm{e}^{i k x}\right)$,

with absorption and emission operators $c_{j}, c_{k}^{\dagger}$ obeying the anti-commutation relations

$\left\{c_{j}(\boldsymbol{k}), c_{k}^{\dagger}\left(\boldsymbol{k}^{\prime}\right)\right\}=\delta_{j k} \delta^{(3)}\left(\boldsymbol{k}-\boldsymbol{k}^{\prime}\right)$.
Conventionally, the $\tilde{u}$-field is called an anti-ghost field. The absorption and emission parts with the adjoint operators will be indexed by \pm -signs below again. They satisfy the following anti-commutation relations:

$$
\begin{aligned}
& \left\{u^{-}(x), \tilde{u}^{+}(y)\right\} \\
& =(2 \pi)^{-3} \int \frac{\mathrm{d}^{3} k}{2 \omega(\boldsymbol{k})} \mathrm{e}^{-i k(x-y)}=i \Delta^{+}(x-y), \\
& \left\{u^{+}(x), \tilde{u}^{-}(y)\right\} \\
& =-(2 \pi)^{-3} \int \frac{\mathrm{d}^{3} k}{2 \omega(\boldsymbol{k})} \mathrm{e}^{i k(x-y)}=i \Delta^{-}(x-y) .
\end{aligned}
$$

All other anti-commutators vanish. This implies

$\{u(x), \tilde{u}(y)\}=i \Delta(x-y)$

and $\{u(x), u(y)\}=0$. Still the nilpotent gauge charge $Q$ satisfying Eq. (113) is given by

$Q=\int \mathrm{d}^{3} x\left[\partial_{\nu} A^{\nu} \partial_{0} u-\left(\partial_{0} \partial_{\nu} A^{\nu}\right) u\right] \stackrel{\text { def }}{=} \int \mathrm{d}^{3} x \partial_{\nu} A^{\nu} \stackrel{\leftrightarrow}{\partial}_{0} u$

where the integrals are taken over any plane $x^{0}=$ const.

Using the Leibnitz rule $\{A B, C\}=A\{B, C\}-[A, C] B$ for graded algebras for the present gauge charge for massless spin-1 fields

$$
\begin{aligned}
Q^{2}= & \frac{1}{2}\{Q, Q\}=\frac{1}{2} \int_{x_{0}=\text { const. }} \mathrm{d}^{3} x \partial_{\nu} A^{\nu}(x)\left\{\stackrel{\leftrightarrow}{\partial}_{0} u(x), Q\right\} \\
& -\frac{1}{2} \int_{x_{0}=\text { const. }} \mathrm{d}^{3} x\left[\partial_{\nu} A^{\nu}(x), Q\right] \stackrel{\leftrightarrow}{\partial}_{0} u(x)=0
\end{aligned}
$$

together with the facts that $\{u(x), u(y)\}=0$ and

$$
\left[\partial_{v} A^{v}(x), Q\right]=-i \partial_{v} \partial^{v} u(x)=0
$$

finally shows that $Q$ is nilpotent.

On the ghost sector, the Krein structure is introduced by

$c_{2}^{K}(\boldsymbol{k})=c_{1}^{\dagger}(\boldsymbol{k}), \quad c_{1}^{K}(\boldsymbol{k})=c_{2}^{\dagger}(\boldsymbol{k})$,

so that $u^{K}=u$ is $K$-self-adjoint and $\tilde{u}^{K}=-\tilde{u}$. Then $Q$ is densely defined on the Fock-Hilbert space and becomes $K$ symmetric $Q \subset Q^{K}$. Roughly speaking, the K-conjugation is the natural generalization of the usual hermitian conjugation to the full (unphysical) Fock space $\mathcal{F}$ which contains time-like and longitudinal photons as well as the fermionic ghost states. Again, positivity on the Fock-Hilbert space can only be maintained by the introduction of the Krein structure. Enforcing $K=\dagger$ would necessitate the existence of negative norm states in the ghost sector. The strategy preferred here is based on a true Hilbert space approach. 
It is convenient to introduce bosonic operators which destroy or create unphysical photon states which are a combination of time-like and longitudinal states

$b_{1,2}=\left(a_{\|} \pm a_{0}\right) / \sqrt{2}, \quad a_{\|}=k_{j} a^{j} /|\boldsymbol{k}|$,

satisfying ordinary commutation relations

$\left[b_{i}(\boldsymbol{k}), b_{j}^{\dagger}(\boldsymbol{k})\right]=\delta_{i j} \delta^{(3)}\left(\boldsymbol{k}-\boldsymbol{k}^{\prime}\right)$

Then, the gauge charge $Q$ itself is given by

$Q=\sqrt{2} \int \mathrm{d}^{3} k \omega(\boldsymbol{k})\left[b_{2}^{\dagger}(\boldsymbol{k}) c_{2}(\boldsymbol{k})+c_{1}^{\dagger}(\boldsymbol{k}) b_{1}(\boldsymbol{k})\right]$.

The explicit form of the gauge charge reveals that it generates a transformation where unphysical photon states are transformed into ghost states and vice versa. The transverse physical photon states remain unaffected by a gauge transformation.

A calculation using the decomposition of the anticommutator

$$
\begin{aligned}
& \left\{b_{1}^{\dagger}(\boldsymbol{k}) c_{1}(\boldsymbol{k}), c_{1}^{\dagger}\left(\boldsymbol{k}^{\prime}\right) b_{1}\left(\boldsymbol{k}^{\prime}\right)\right\} \\
& \quad=b_{1}^{\dagger}(\boldsymbol{k})\left\{c_{1}(\boldsymbol{k}), c_{1}^{\dagger}\left(\boldsymbol{k}^{\prime}\right)\right\} b_{1}\left(\boldsymbol{k}^{\prime}\right)-\left[b_{1}^{\dagger}(\boldsymbol{k}), c_{1}^{\dagger}\left(\boldsymbol{k}^{\prime}\right) b_{1}\left(\boldsymbol{k}^{\prime}\right)\right] c_{1}(\boldsymbol{k}) \\
& =\left(b_{1}^{\dagger}(\boldsymbol{k}) b_{1}(\boldsymbol{k})+c_{1}^{\dagger}(\boldsymbol{k}) c_{1}(\boldsymbol{k})\right) \delta^{(3)}\left(\boldsymbol{k}-\boldsymbol{k}^{\prime}\right)
\end{aligned}
$$

shows that the anti-commutator

$$
\begin{aligned}
\left\{Q^{\dagger}, Q\right\}=2 & \int \mathrm{d}^{3} k \boldsymbol{k}^{2}\left[b_{1}^{\dagger}(\boldsymbol{k}) b_{1}(\boldsymbol{k})\right. \\
& \left.+b_{2}^{\dagger}(\boldsymbol{k}) b_{2}(\boldsymbol{k})+c_{1}^{\dagger}(\boldsymbol{k}) c_{1}(\boldsymbol{k})+c_{2}^{\dagger}(\boldsymbol{k}) c_{2}(\boldsymbol{k})\right]
\end{aligned}
$$

is essentially the number operator for unphysical particles apart from the phase space factor $\omega(\boldsymbol{k})^{2}=\boldsymbol{k}^{2}$. Therefore, if a state $|\Phi\rangle$ in the Fock-Hilbert space satisfies $\left\{Q^{\dagger}, Q\right\}|\Phi\rangle=0$, it contains physical transverse photon states only. Hence, the physical Hilbert space is the kernel

$\mathcal{F}_{\text {phys }}=\operatorname{Ker}\left\{Q^{\dagger}, Q\right\}$.

Additionally, since $\left\{Q^{\dagger}, Q\right\}=Q^{\dagger} Q+Q Q^{\dagger}$ is self-adjoint and positive

$$
\left\langle\Phi \mid\left(Q^{\dagger} Q+Q Q^{\dagger}\right) \Phi\right\rangle=\|Q \Psi\|^{2}+\left\|Q^{\dagger} \Phi\right\|^{2} \geq 0 .
$$

This expression vanishes only iff $Q \Phi=Q^{\dagger} \Phi=0$, leading to another characterization of the physical Hilbert space

$\mathcal{F}_{\text {phys }}=\operatorname{Ker} Q \cap \operatorname{Ker} Q^{\dagger}$.
Ker $Q$ is a subspace of $\mathcal{F}$ and orthogonal to the closure $\overline{\operatorname{Ran} \mathrm{Q}^{\dagger}}$ of the range of $Q^{\dagger}$, since for $|\Phi\rangle \in \operatorname{Ker} Q$ one has

$\langle Q \Phi \mid \Psi\rangle=0=\left\langle\Phi \mid Q^{\dagger} \Psi\right\rangle$

In fact, $\mathcal{F}$ has the direct decomposition

$\mathcal{F}=\operatorname{Ker} Q \oplus \overline{\operatorname{Ran} Q^{\dagger}}=\operatorname{Ker} Q^{\dagger} \oplus \overline{\operatorname{Ran} \mathrm{Q}}$.

This can be proven by noticing that the domain $\operatorname{Dom}\left(Q^{\dagger}\right)$ is dense in $\mathcal{F}$, so if $\left\langle\Upsilon \mid Q^{\dagger} \Psi\right\rangle=0$ for all $\Psi \in \operatorname{Dom}\left(Q^{\dagger}\right)$, then $\langle Q \Upsilon \mid \Psi\rangle=0$, implying $|Q \Upsilon\rangle=0$ or $|\Upsilon\rangle \in \operatorname{Ker} Q$. Using the nilpotency $Q^{2}=0$ one sees from $\left\langle Q^{\dagger} \Psi \mid Q \Phi\right\rangle=$ $\left\langle\Psi \mid Q^{2} \Phi\right\rangle 0=$ that $\overline{\operatorname{Ran} \mathrm{Q}^{\dagger}}$ is orthogonal to $\overline{\operatorname{Ran} \mathrm{Q}}$. Consequently, $\mathcal{F}$ has the direct decomposition

$\mathcal{F}=\overline{\operatorname{Ran} \mathrm{Q}^{\dagger}} \oplus \overline{\operatorname{Ran} \mathrm{Q}} \oplus \mathcal{F}_{\text {phys }}$.

Indeed, if $P_{1}$ and $P_{2}$ are projection operators on the first two subspaces above, due to orthogonality one has $P_{1} P_{2}=0=$ $P_{2} P_{1}$. It follows that the projection operator on the orthogonal complement of $P_{1,2}$ is given by

$1-\left(P_{1}+P_{2}\right)=\left(1-P_{1}\right)\left(1-P_{2}\right)$

which is the projection onto $\operatorname{Ker} Q \cap \operatorname{Ker} Q^{\dagger}$, the physical subspace. Obviously,

Ker $Q=\mathcal{F}_{\text {phys }} \oplus \overline{\operatorname{Ran} \mathrm{Q}}$,

accordingly

$\mathcal{F}_{\text {phys }}=\operatorname{Ker} Q / \overline{\operatorname{Ran} \mathrm{Q}}$.

One may note that $\operatorname{Ran} \mathrm{Q}=\operatorname{Dom}\left(Q^{-1}\right)$ is indeed not closed since $Q^{-1}$ is unbounded for a massless gauge field $A^{\mu}$.

Returning to the defining property of $Q$ as being the infinitesimal generator of gauge transformations given by Eq. (111) and Eq. (113), the notation

$d_{Q} F=[Q, F]$,

if the (normally ordered) product of free fields $F$ contains only bosonic fields and an even number of ghost fields, and

$d_{Q} F=\{Q, F\}=Q F+F Q$,

if $F$ contains an odd number of ghost fields, may be introduced for practical reasons. Then $d_{Q}$ has all properties of an anti-derivation, in particular the identity

$\{A B, C\}=A\{B, C\}-[A, C] B$ 
implies the product rule

$d_{Q}(F(x) G(y))=\left(d_{Q} F(x)\right) G(y)+(-1)^{n_{F}} F(x) d_{Q} G(y)$,

where $n_{F}$ is the ghost number of $F$, i.e. the number of $u$ 's in $F$ minus the number of $\tilde{u}$-fields. The gauge variations $d_{Q}$ of some free fields now are

$d_{Q} A^{\mu}=i \partial^{\mu} u, \quad d_{Q} A_{ \pm}^{\mu}=i \partial^{\mu} u_{ \pm}, \quad d_{Q} u=0$,

$d_{Q} \tilde{u}=\{Q, \tilde{u}\}=-i \partial_{\mu} A^{\mu}, \quad d_{Q} \tilde{u}_{ \pm}=-i \partial_{\mu} A_{ \pm}^{\mu}$.

The latter follows from the anti-commutation relation Eq. (137). $d_{Q}$ changes the ghost number by one, i.e. a bosonic field goes over into a fermionic field and vice versa. Then the nilpotency $Q^{2}=0$ implies for a bosonic field $F_{B}$

$$
\begin{aligned}
d_{Q}^{2} F_{B} & =\left\{Q,\left[Q, F_{B}\right]\right\} \\
& =Q\left(Q F_{B}-F_{B} Q\right)+\left(Q F_{B}-F_{B} Q\right) Q=0,
\end{aligned}
$$

and for a Fermi field $F$

$$
\begin{aligned}
d_{Q}^{2} F & =[Q,\{Q, F\}] \\
& =Q\left(Q F_{B}+F_{B} Q\right)-\left(Q F_{B}-F_{B} Q\right) Q=0,
\end{aligned}
$$

hence

$d_{Q}^{2}=0$

is also nilpotent. For such situations one can use notions from homological algebra, for example, if

$F=d_{Q} G$,

the $F$ is called a coboundary [13]. The gauge variation $d_{Q}$ has some similarity with the BRST transformation in the functional approach to QCD. However, the BRST transformation operates on interacting fields (mainly classical) and the quantum gauge invariance which will be defined below for free fields displays some technical differences compared to BRST invariance [14].

To end this section, the operator gauge transformation when working with fermionic ghosts shall be considered. It is straightforward to see that the Lie series

$\mathrm{e}^{-i \lambda Q} A^{\mu} \mathrm{e}^{+i \lambda Q}=A^{\mu}-\frac{i \lambda}{1 !}\left[Q, A^{\mu}\right]-\frac{\lambda^{2}}{2 !}\left[Q,\left[Q, A^{\mu}\right]\right]+\cdots$

terminates after the second-order term. Since

$$
\begin{aligned}
{[Q, u(x)] } & =Q u(x)-u(x) Q=\{Q, u(x)\}-2 u(x) Q \\
& =-2 u(x) Q,
\end{aligned}
$$

one has

$[Q, u(x) Q]=[Q, u(x)] Q+u(x)[Q, Q]=0$,

or, stated equivalently

$$
\begin{aligned}
{[Q, u(x) Q] } & =Q u(x) Q-u(x) Q^{2} \\
& =Q u(x) Q+u(x) Q^{2}=\{Q, u(x)\} Q=0 .
\end{aligned}
$$

Consequently, the gauge transformation of the gauge potential is found to be given by

$$
\begin{aligned}
A^{\prime \mu}(x) & =A^{\mu}(x)+\lambda \partial^{\mu} u(x)+i \lambda^{2} \partial^{\mu} u(x) Q \\
& =A^{\mu}(x)+\partial^{\mu} u(x)\left(\lambda+i \lambda^{2} Q\right) .
\end{aligned}
$$

Analogously, one finds for the ghost fields

$u^{\prime}(x)=u(x)+2 i \lambda u(x) Q$,

$\tilde{u}^{\prime}(x)=\tilde{u}(x)+\lambda\left(2 i \tilde{u}(x) Q-\partial_{\mu} A^{\mu}(x)\right)-i \lambda^{2} \partial_{\mu} A^{\mu}(x) Q$.

5.2 Definition of perturbative quantum gauge invariance

We take the next step towards full QED and couple photons to electrons. In perturbative QED, the $S$-matrix is expanded as a power series in the coupling constant $e$. At first order, the interaction is described by the normally ordered product of free fields

$\mathcal{H}_{\text {int }}(x)=-\mathcal{L}_{\text {int }}^{\mathrm{QED}}(x)=-\mathrm{e}: \bar{\Psi}(x) \gamma^{\mu} \Psi(x): A_{\mu}(x)$,

where $\Psi$ is the electron field operator and $\mathrm{e}=-e>0$ the elementary charge. The $S$-matrix is then usually given in the literature by the formal expression ( $T$ denotes time ordering)

$$
\begin{aligned}
S & =\mathbf{1}+\sum_{n=1}^{\infty} \frac{(-i)^{n}}{n !} \int_{\mathbb{R}^{4 n}} \mathrm{~d}^{4} x_{1} \ldots \mathrm{d}^{4} x_{n} T\left[\mathcal{H}_{\text {int }}\left(x_{1}\right) \ldots \mathcal{H}_{\text {int }}\left(x_{n}\right)\right] \\
& =\mathbf{1}+\sum_{n=1}^{\infty} \frac{1}{n !} \int \mathrm{d}^{4} x_{1} \ldots \mathrm{d}^{4} x_{n} T_{n}\left(x_{1}, \ldots x_{n}\right)
\end{aligned}
$$

where we have introduced the time-ordered products $T_{n}$ for notational simplicity, and we have

$$
T_{1}(x)=-i \mathcal{H}_{\text {int }}(x)=i \mathrm{e}: \bar{\Psi}(x) \gamma^{\mu} \Psi(x): A_{\mu}(x) .
$$

Expression (174) is plagued by infrared and ultraviolet divergences. We leave this technical problem aside and we assume that the $T_{n}$ are already regularized, well-defined operatorvalued distributions, which are symmetric in the space coordinates $\left(x_{1}, \ldots, x_{n}\right)$.

A precise definition of perturbative quantum gauge invariance for QED, which works in a very analogous way for $\mathrm{QCD}$, can be derived by investigating how infinitesimal 
gauge transformations act on the higher orders of the perturbative $S$-matrix. One considers the (anti-)commutators

$$
\begin{aligned}
{\left[Q, A_{\mu}\right] } & =i \partial_{\mu} u, \quad\{Q, u\}=0, \quad\{Q, \tilde{u}\}=-i \partial_{\nu} A^{v}, \\
{[Q, \Psi] } & =[Q, \bar{\Psi}]=0 .
\end{aligned}
$$

The commutators of $Q$ with the electron field are of course trivial, since the operators act on different Fock space sectors. Only the first and the last two commutators in Eq. (176) are needed here, the others would become important in QCD. Note, however, that ordinary commutation relations of the electron field with $Q$ or the ghost fields $u$ and $\tilde{u}$ can be switched into anti-commutation relations by a Klein transformation (see [15] and references therein) without changing the physical content of the theory. From Eq. (131) one knows that the commutator of $Q$ with an operator gives the first-order variation of the operator subject to a gauge transformation. Then, for the first-order interaction $T_{1}$

$$
\begin{aligned}
{\left[Q, T_{1}(x)\right] } & =-\mathrm{e}: \bar{\Psi}(x) \gamma^{\mu} \Psi(x): \partial_{\mu} u(x) \\
& =i \partial_{\mu}\left(i \mathrm{e}: \bar{\Psi}(x) \gamma^{\mu} \Psi(x): u(x)\right)=i \partial_{\mu} T_{1 / 1}^{\mu}(x) .
\end{aligned}
$$

Here, electron current conservation was used

$\partial_{\mu}: \bar{\Psi} \gamma^{\mu} \Psi:=0$.

Note that the free electron field is not affected by the gauge transformation. The term

$T_{1 / 1}^{\mu}=i \mathrm{e}: \bar{\Psi} \gamma^{\mu} \Psi: u$,

called the 'Q-vertex' or 'gauge vertex' of QED, can be used in a generalized manner from the first-order equation (177) to the $n$ th-order:

$$
\begin{aligned}
{\left[Q, T_{n}\left(x_{1}, \ldots x_{n}\right)\right] } & =i \sum_{l=1}^{n} \partial_{\mu}^{x_{l}} T_{n / l}^{\mu}\left(x_{1}, \ldots x_{n}\right) \\
& =\text { (sum of divergences }),
\end{aligned}
$$

where $T_{n / l}^{\mu}$ is again a mathematically well-defined version of the time-ordered product

$T_{n / l}^{\mu}\left(x_{1}, \ldots, x_{n}\right){ }^{\prime}={ }^{\prime} T\left(T_{1}\left(x_{1}\right) \ldots T_{1 / 1}^{\mu}\left(x_{l}\right) \ldots T_{1}\left(x_{n}\right)\right)$,

thereby defining by Eq. (180) the condition of gauge invariance in QED [16].

If one considers for a fixed $x_{l}$ all terms in $T_{n}$ with the external field operator $A_{\mu}\left(x_{l}\right)$

$T_{n}\left(x_{1}, \ldots x_{n}\right)=: t_{l}^{\mu}\left(x_{1}, \ldots x_{n}\right) A_{\mu}\left(x_{l}\right):+\cdots$ (the dots represent terms without $A_{\mu}\left(x_{l}\right)$ ), then gauge invariance Eq. (180) requires

$\partial_{\mu}^{l}\left[t_{l}^{\mu}\left(x_{1}, \ldots x_{n}\right) u\left(x_{l}\right)\right]=t_{l}^{\mu}\left(x_{1}, \ldots x_{n}\right) \partial_{\mu} u\left(x_{l}\right)$

or

$\partial_{\mu}^{l} t_{l}^{\mu}\left(x_{1}, \ldots x_{n}\right)=0$

i.e. one obtains the Ward-Takahashi identities [17] for QED. The Ward-Takahashi identities express the implications of gauge invariance of QED, which is defined here on the operator level, by C-number identities for Green's distributions.

The main property of gauge invariance of perturbative QED can be stated as follows: there exists a symmetry transformation generated by the gauge charge $Q$, which leaves the $S$-matrix elements invariant, since the gauge transformation only adds divergences in the analytic sense to the $S$-matrix expansion which vanish after integration over the coordinates $x_{1}, \ldots x_{n}$.

The observation that QED is gauge invariant is interesting on its own, but the true importance of gauge invariance is the fact that it allows one to prove on a formal level the unitarity of the $S$-matrix on the physical subspace ([16]. Due to the presence of the skew-adjoint operator $A^{0}$ in the firstorder coupling term Eq. (173) which defines the interaction between fermions and gauge fields or the related presence of unphysical ghost and longitudinal and time-like photon states in QED formulated in a local and renormalizable gauge, the $S$-matrix is not unitary on the full Fock space, but it is on $\mathcal{F}_{\text {phys. }}$ An full algebraic proof shall not be given here, but we emphasize that gauge invariance is the basic prerequisite which ensures unitarity, a fact which becomes plausible when one assures oneself that a gauge transformation acts only on the unphysical sector of a gauge theory. A detailed discussion of this fact can be found in $[11,16,18]$. Ghosts are introduced only as a formal but convenient tool, they 'blow up' the Fock space and they do not interact with the electrons and photons. In QCD, the situation is far more complicated than in QED when non-perturbative aspects of the theory have to be considered.

The perturbative expression Eq. (174) is problematic, because the time-ordered products $T_{n}$ are operator-valued distributions after regularization, and they have to be smeared out by test functions. In order to be more precise in the mathematical sense, one has to introduce a test function $g_{0}(x) \in \mathcal{S}\left(\mathbf{R}^{4}\right)$ normalized such that $g_{0}(0)=1$ and replace expression Eq. (174) by

$$
S=\mathbf{1}+\sum_{n=1}^{\infty} \frac{1}{n !} \int \mathrm{d}^{4} x_{1} \ldots \mathrm{d}^{4} x_{n} T_{n}\left(x_{1}, \ldots x_{n}\right) g_{0}\left(x_{1}\right) \ldots g_{0}\left(x_{n}\right) .
$$


Here, $g_{0}$ acts as an infrared regulator, which switches off the long range part of the interaction in theories where massless fields are involved. E.g., in QED the emission of soft photons is switched by $g_{0}$, and as long as the so-called adiabatic limit $g_{0} \rightarrow 1$ has not been performed, $S$-matrix elements remain finite. One possibility to perform the adiabatic limit is by scaling the switching function $g_{0}(x)$, i.e. one replaces $g_{0}(x)$ by $g(x)=g_{0}(\epsilon x)$ and performs the limit $\epsilon \rightarrow 0$, such that $g$ and the coupling strength everywhere approaches a constant value. If the $S$-matrix is modified by a gauge transformation, operators which are divergences are added to the $n$ th-order term $T_{n}$. Such a contribution can be written as

$$
\begin{aligned}
\int \mathrm{d}^{4} & x_{1} \ldots \mathrm{d}^{4} x_{n} \\
& \times \partial_{\mu}^{x_{l}} O^{\ldots \mu \ldots}\left(x_{1}, \ldots, x_{l}, \ldots x_{n}\right) g\left(x_{1}\right) \ldots g\left(x_{l}\right) \ldots g\left(x_{n}\right) \\
= & -\int \mathrm{d}^{4} x_{1} \ldots \mathrm{d}^{4} x_{n} \\
& \times O^{\ldots \mu \ldots}\left(x_{1}, \ldots, x_{l}, \ldots x_{n}\right) g\left(x_{1}\right) \ldots \partial_{\mu}^{x_{l}} g\left(x_{l}\right) \ldots g\left(x_{n}\right) .
\end{aligned}
$$

In the adiabatic limit, the gradient $\partial_{\mu}^{x_{l}} g\left(x_{l}\right)$ vanishes. Unfortunately, this property of the scaling limit does not guarantee that the whole term Eq. (186) vanishes. Introducing a switching function $g_{0}$ is the natural infrared regularization in the framework of operator-valued distributions, but it destroys the Poincaré invariance of the theory and leads to a problem to define the physical vacuum. Whereas this problem more or less might be under control for QED, it is a serious problem expressed by the catchwords 'infrared slavery' for QCD. The infrared problem is not really understood in QCD, and all proofs of unitarity which exist in the literature have to be taken with a grain of salt, because they avoid the discussion of infrared problems somehow.

The fermionic derivative coupling model defined in the following section emerges as a special limit when one considers perturbative QED with a vanishing coupling constant $e$, maintaining only an unphysical part of the interaction.

\subsection{The model}

Starting from the field equations again, keeping in mind that one has to take care of the order of products in the case of fermionic fields, one has

$$
\begin{aligned}
& \left(i \gamma_{\mu} \partial^{\mu}-m\right) \psi(x)=g \partial^{\mu} \varphi(x) \gamma_{\mu} \psi(x), \\
& \square \varphi(x)=0, \\
& \square A^{\mu}(x)=0 .
\end{aligned}
$$

The gauge field $A^{\mu}(x)$ is rather an additional spectator. The coupling term in Eq. (187) emerges when considering a gauge transformed version of the first-order coupling term in QED given by Eq. (175) according to Eq. (170), in the limit where $e \rightarrow 0$ but $e \lambda^{2}=g$ held fixed.
We define $\varphi(x)=-i Q u(x)$ with the help of the gauge charge operator given in Eq. (144) and use the fermionic scalar field with the properties displayed by Eqs. (132)-(137). An operator solution of the equation of motion above then reads

$$
\begin{aligned}
\psi(x) & =\mathrm{e}^{-i g \varphi(x)} \psi_{0}(x) \\
& =\left[1-g Q u(x)+g^{2} Q u(x) Q u(x)+\cdots\right] \psi_{0}(x) \\
& =\left[1-g Q u(x)-g^{2} Q^{2} u(x) u(x)-\cdots\right] \psi_{0}(x) \\
& =[1-i g \varphi(x)] \psi_{0}(x) .
\end{aligned}
$$

Here we use the free fields $A^{\mu}(x)$ and $\psi_{0}(x)$ acting on the Fock-Hilbert space introduced in the discussion of QED, satisfying

$A^{\mu}(x)=0, \quad\left(i \gamma_{\mu} \partial^{\mu}-m\right) \psi_{0}(x)=0$,

and $\varphi(x)$ satisfying the commutation relation

$$
\begin{aligned}
{[\varphi(x), \varphi(y)] } & =-[Q u(x), Q u(y)] \\
& =-Q u(x) Q u(y)+Q u(y) Q u(x) \\
& =Q^{2} u(x) u(y)-Q^{2} u(y) u(x)=0 .
\end{aligned}
$$

Since $\{Q, u\}=0$, we have $Q u=-u Q$. Additionally, $Q$ is nilpotent, $Q^{2}=0 . u$ is an unphysical Fermi field, $u(x) u(x)=: u(x) u(x):=-: u(x) u(x):=0$ and : $\partial^{\mu} u(x) u(x):=0$ hold and similar identities hold for $\varphi$, accordingly,

$\psi_{0}(x)=[1+i g \varphi(x)][1-i g \varphi(x)] \psi_{0}(x)=[1+i g \varphi(x)] \psi(x)$.

Inserting the operator solution Eq. (190) into Eq. (187) leads to

$$
\begin{aligned}
& \left(i \gamma_{\mu} \partial^{\mu}-m\right) \psi(x) \\
& \quad=i \gamma_{\mu} \partial^{\mu}\left([1-i g \varphi(x)] \psi_{0}(x)\right)-m \psi(x) \\
& \quad=[1-i g \varphi(x)]\left[i \gamma_{\mu} \partial^{\mu} \psi_{0}(x)-m \psi_{0}(x)\right]+g \partial^{\mu} \varphi(x) \gamma_{\mu} \psi_{0}(x) \\
& \quad=g \partial^{\mu} \varphi(x)[1+i g \varphi(x)] \gamma_{\mu} \psi(x)=g \partial^{\mu} \varphi(x) \gamma_{\mu} \psi(x) .
\end{aligned}
$$

The interaction term is unphysical and gauge invariant in the sense that

$\left[Q, \mathcal{H}_{\text {int }}(x)\right]=-g\left[Q,: \bar{\psi}(x) \gamma^{\mu} \psi(x): \partial_{\mu} \varphi(x)\right]=0$.

$\mathcal{H}_{\text {int }}$ is $\mathrm{K}$-symmetric like $\varphi^{K}=(-i Q u)^{K}=i u^{K} Q^{K}=$ $-i Q u=\varphi$.

The model presented above can be modified in the following way. Let $a(x)$ be a C-number field with $a(0, \boldsymbol{x}) \in \mathcal{S}\left(\mathbb{R}^{3}\right)$ 
satisfying the wave equation $\square a(x)=0$. Then one has the Fourier decompositions

$a(x)=\int \frac{\mathrm{d}^{3} k}{\sqrt{2(2 \pi)^{3} \omega(\boldsymbol{k})}}\left(a_{-}(\boldsymbol{k}) \mathrm{e}^{-i k x}+a_{+}(\boldsymbol{k}) \mathrm{e}^{+i k x}\right)$,

$\partial_{0} a(x)=i \int \mathrm{d}^{3} k \sqrt{\frac{\omega(\boldsymbol{k})}{2(2 \pi)^{3}}}\left(-a_{-}(\boldsymbol{k}) \mathrm{e}^{-i k x}+a_{+}(\boldsymbol{k}) \mathrm{e}^{+i k x}\right)$,

again with $k^{0}=\omega(\boldsymbol{k})=|\boldsymbol{k}|$ and $k x=k^{0} x^{0}-\boldsymbol{k} \boldsymbol{x}$ and analogous Fourier representations hold for the operator-valued distributions $u(x)$ and $\partial_{0} u(x)$.

The definition of the operator

$$
\tilde{Q}=\int_{x_{0}=\text { const. }} \mathrm{d}^{3} x a(x) \stackrel{\leftrightarrow}{\partial_{0}} u(x)
$$

is time-independent; for $x_{0}=0$ one obtains

$$
\begin{aligned}
\tilde{Q}= & \frac{i}{(2 \pi)^{3}} \int \mathrm{d}^{3} x \int \frac{\mathrm{d}^{3} k^{\prime}}{\sqrt{2 \omega\left(\boldsymbol{k}^{\prime}\right)}} \int \mathrm{d}^{3} k \sqrt{\frac{\omega(\boldsymbol{k})}{2}} \\
& \times\left[\left(a_{-}\left(\boldsymbol{k}^{\prime}\right) \mathrm{e}^{i \boldsymbol{k}^{\prime} \boldsymbol{x}}+a_{+}\left(\boldsymbol{k}^{\prime}\right) \mathrm{e}^{-i \boldsymbol{k}^{\prime} \boldsymbol{x}}\right)\right. \\
& \times\left(-c_{2}(\boldsymbol{k}) \mathrm{e}^{i \boldsymbol{k} \boldsymbol{x}}+c_{1}^{\dagger}(\boldsymbol{k}) \mathrm{e}^{-i \boldsymbol{k} \boldsymbol{x}}\right) \\
& -\left(-a_{-}(\boldsymbol{k}) \mathrm{e}^{i \boldsymbol{k} \boldsymbol{x}}+a_{+}(\boldsymbol{k}) \mathrm{e}^{-i \boldsymbol{k} \boldsymbol{x}}\right) \\
& \left.\times\left(c_{2}\left(\boldsymbol{k}^{\prime}\right) \mathrm{e}^{i \boldsymbol{k}^{\prime} \boldsymbol{x}}+c_{1}^{\dagger}\left(\boldsymbol{k}^{\prime}\right) \mathrm{e}^{-i \boldsymbol{k}^{\prime} \boldsymbol{x}}\right)\right] \\
= & \frac{i}{2} \int \mathrm{d}^{3} k\left[-a_{-}(-\boldsymbol{k}) c_{2}(\boldsymbol{k})-a_{+}(\boldsymbol{k}) c_{2}(\boldsymbol{k})\right. \\
& +a_{-}(-\boldsymbol{k}) c_{2}(\boldsymbol{k})-a_{+}(\boldsymbol{k}) c_{2}(\boldsymbol{k}) \\
& +a_{-}(\boldsymbol{k}) c_{1}^{\dagger}(\boldsymbol{k})+a_{+}(-\boldsymbol{k}) c_{1}^{\dagger}(\boldsymbol{k}) \\
& \left.+a_{-}(\boldsymbol{k}) c_{1}^{\dagger}(\boldsymbol{k})-a_{+}(-\boldsymbol{k}) c_{1}^{\dagger}(\boldsymbol{k})\right] \\
= & i \int \mathrm{d}^{3} k\left[-a_{+}(\boldsymbol{k}) c_{2}(\boldsymbol{k})+a_{-}(\boldsymbol{k}) c_{1}^{\dagger}(\boldsymbol{k})\right] .
\end{aligned}
$$

Again one has $\tilde{Q}^{2}=\frac{1}{2}\{\tilde{Q}, \tilde{Q}\}=0$, therefore the model discussed above can be formulated with $\tilde{Q}$ instead of $Q$ without a quantized vector field $A^{\mu}$ when $a_{-}^{*}(\boldsymbol{k})=a_{+}(\boldsymbol{k})$ is invoked, i.e. $a(x)$ must be real. Then $Q$ becomes $K$-symmetric, since

$Q^{K}=i \int \mathrm{d}^{3} k\left[-a_{-}^{*}(\boldsymbol{k}) c_{2}(\boldsymbol{k})+a_{+}^{*}(\boldsymbol{k}) c_{1}^{\dagger}(\boldsymbol{k})\right]$

and the Krein correlator of the $\psi$-field remains trivial,

$\left\langle 0\left|\psi_{0}(x) \bar{\psi}_{0}(y)\right| 0\right\rangle=\left\langle 0\left|\psi_{0}(x) \psi_{0}^{K}(y)\right| 0\right\rangle=\left\langle 0\left|\psi(x) \psi^{K}(y)\right| 0\right\rangle$.
However, since

$\left\{\tilde{Q}^{\dagger}, Q\right\}=\int \mathrm{d}^{3} k\left(\left|a_{-}(\boldsymbol{k})\right|^{2}+\left|a_{+}(\boldsymbol{k})\right|^{2}\right)$,

the original specification of the physical space according to Eq. (147) is lost. It is left to the reader to couple the ghost field $u$ instead of $\varphi$ to $\psi$ in the same way as a simple exercise.

The fermionic model is physically trivial, the formalism rather involved, but at the same time we have one possible variant of the classical derivative coupling model which served here for the introduction of the concepts related to the operator gauge formalism. Non-renormalizable expressions or non-tempered distributions nowhere appear, despite the dimension of the coupling term.

\section{Conclusions}

The two models presented in this work are a tool to demonstrate the fact that there are several ways to quantize a classical field theory. The models also clarify that the rôle of the fields is rather to implement the principle of causality, but the type and number of the fields appearing in a theory is rather unrelated to the physical spectrum of empirically observable particles. The fields are coordinatizations of an underlying physical theory and carriers of charges which finally serve to extract the algebra of the observables.

From a distributional point of view, theories based on point-like localized quantum fields may indicate that the frame of Schwartz operator-valued distributions favored in perturbative QFT is too narrow, but it remains unclear whether a loss of the original concepts using tempered distributions can be avoided within a suitable formalism.

Open Access This article is distributed under the terms of the Creative Commons Attribution License which permits any use, distribution, and reproduction in any medium, provided the original author(s) and the source are credited.

Article funded by SCOAP $^{3}$ and licensed under CC BY 4.0

\section{Appendix A: A distributive toolbox}

\section{A.1 Support}

A distribution $d \in \mathcal{S}^{\prime}\left(\mathbb{R}^{n}\right)$ is called regular, if it can be represented by

$d(f)=\int_{\mathbb{R}^{n}} \mathrm{~d} x d(x) f(x)$,

where $d(x)$ is a locally integrable function and $f \in \mathcal{S}\left(\mathbb{R}^{n}\right)$. This close analogy between functions and distributions leads 
to the definition of the support of distributions. The support of a function defined on $\mathbb{R}^{n}$ is the closure of the set where the function is non-zero,

$\operatorname{supp}(f)=\overline{\left\{x \in \mathbb{R}^{n} \mid f(x) \neq 0\right\}}$.

A point $x$ belongs to the support of a distribution $d$ iff for every neighborhood $U_{x}$ of $x$ a function $f$ exists with $\operatorname{supp}(f) \subset U_{x}$ and $d(f) \neq 0$.

\section{A.2 Tensor product of distributions}

Let $d_{1} \in \mathcal{S}^{\prime}\left(\mathbb{R}^{n}\right), d_{2} \in \mathcal{S}^{\prime}\left(\mathbb{R}^{m}\right)$. Then a unique distribution $h \in \mathcal{S}^{\prime}\left(\mathbb{R}^{n+m}\right)$ exists such that for all $f_{1}(x) \in \mathcal{S}\left(\mathbb{R}^{n}\right)$, $f_{2}(y) \in \mathcal{S}\left(\mathbb{R}^{m}\right)$

$h\left(f_{1}(x) f_{2}(y)\right)=d_{1}\left(f_{1}(x)\right) d_{2}\left(f_{2}(y)\right)$.

$h=d_{1} \otimes d_{2}$ is the tensor product of $d_{1}$ and $d_{2}$. A simple example is given by the product of Dirac distributions

$\delta^{(n)}(x)=\delta\left(x^{1}\right) \delta\left(x^{2}\right) \ldots \delta\left(x^{n}\right), \quad x=\left(x^{1}, x^{2}, \ldots, x^{n}\right)$,

where

$\int_{\mathbb{R}^{n}} \mathrm{~d}^{n} x \delta^{(n)}(x) f(x)=f(0)$.

The Fourier transform of the above distribution is given by

$$
\begin{aligned}
& \hat{\delta}^{(n)}(k)=(2 \pi)^{-n / 2}, \\
& \int_{\mathbb{R}^{n}} \mathrm{~d}^{n} x \mathrm{e}^{i k^{1} x^{1}+\ldots+i k^{n} x^{n}}=(2 \pi)^{n} \delta^{(n)}(k) .
\end{aligned}
$$

In close analogy, tensor products of free fields, e.g., the products of two scalar fields on $\mathbb{R}^{4}$ like $\varphi(x) \varphi(y)$, are again operator-valued distributions, in the present case on $\mathbb{R}^{8}$. However, products like $\delta(x) \delta(x)$ (or $\left.\varphi(x) \varphi(x)\right)$ are illdefined, but they can be regularized (by normal ordering) in order to have well-defined (operator-valued) distributions.

\section{A.3 Principal values and regularization}

An important distribution is $P \frac{1}{x}$, i.e. the principal value of the singular function $1 / x \in C(\mathbb{R} \backslash 0)$ interpreted as a distribution:

$$
P \frac{1}{x}(f)=\lim _{\varepsilon \searrow 0} \int_{|x|>\varepsilon} \mathrm{d} x \frac{f(x)}{x}, \quad P \frac{1}{x}=\frac{\mathrm{d}}{\mathrm{d} x} \ln |x| .
$$

$P \frac{1}{x}$ is a regularization of the divergent expression $\frac{1}{x}$. Without regularization, $1 / x$ is only defined on

$\mathcal{S}_{0}(\mathbb{R})=\{f \in \mathcal{S}(\mathbb{R}) \mid f(0)=0\}$,

where the singular behavior of $1 / x$ at $x=0$ gets absorbed. $P \frac{1}{x}$ can be viewed as an extension of $\left.\frac{1}{x}\right|_{\mathcal{S}_{0}(\mathbb{R})}$ to the whole test function space $\mathcal{S}(\mathbb{R})$ according to the Hahn-Banach theorem. One may also write

$P \frac{1}{x}(f)=\int_{0}^{\infty} \mathrm{d} x \frac{f(x)-f(-x)}{x}$

A canonical regularization of the divergent, nonregularized integral

$d_{1 / x^{2}}^{\mathrm{nr}}(f)=\int_{\mathbb{R}} \mathrm{d} x \frac{f(x)}{x^{2}}$

is possible by shifting the derivative

$d_{1 / x^{2}}(f)=\int_{\mathbb{R}} \mathrm{d} x P \frac{1}{x} f^{\prime}(x)$.

Equivalently, one may regularize

$\left(x^{-2}, f\right)_{\text {reg }}=\int_{0}^{\infty} \mathrm{d} x \frac{f(x)+f(-x)-2 f(0)}{x^{2}}$.

\section{A.4 Renormalization}

In regularization procedures, a distribution declared by a divergent expression becomes properly redefined within a range of permissible solutions allowed by physical conditions. Subsequent renormalizations within this range then may be performed. It is often exploited that certain distributions exhibit a specific scaling behavior. E.g., the renormalization

$d_{1 / x^{2}} \rightarrow d_{1 / x^{2}}+C \cdot \delta^{\prime}(x)$

respects the scaling behavior $(\lambda>0)$ of the distribution $d_{1 / x^{2}}$, because

$$
\begin{aligned}
\delta^{\prime}(f) & =-f^{\prime}(0) \stackrel{\text { formally }}{=} \int_{\mathbb{R}} \mathrm{d} x \delta^{\prime}(x) f(x) \\
& =-\int_{\mathbb{R}} \mathrm{d} x \delta(x) f^{\prime}(x)
\end{aligned}
$$


scales as

$$
\begin{aligned}
\int_{\mathbb{R}} \mathrm{d} x \delta^{\prime}(\lambda x) f(x) \stackrel{x^{\prime}}{=}=\lambda x & \int_{\mathbb{R}} \frac{\mathrm{d} x^{\prime}}{\lambda} \delta^{\prime}\left(x^{\prime}\right) f\left(x^{\prime} / \lambda\right) \\
& =-\int_{\mathbb{R}} \frac{\mathrm{d} x^{\prime}}{\lambda^{2}} \delta\left(x^{\prime}\right) f^{\prime}\left(x^{\prime} / \lambda\right)=-\frac{1}{\lambda^{2}} f^{\prime}(0),
\end{aligned}
$$

i.e.

$$
\delta^{\prime}(\lambda x)=\lambda^{-2} \delta^{\prime}(x)
$$

and

$d_{1 / x^{2}}(\lambda x)=(\lambda x)_{\mathrm{reg}}^{-2}=\lambda^{-2} d_{1 / x^{2}}(x)$.

A.5 Sokhotsky-Plemelj formula

The distributions

$$
\frac{1}{x \pm i 0}=P \frac{1}{x} \mp i \pi \delta(x)
$$

are often constructed from a limiting procedure,

$$
\int_{\mathbb{R}} \frac{f(x)}{x+i 0} \mathrm{~d} x=\lim _{\varepsilon \searrow 0} \int_{\mathbb{R}} \frac{f(x)}{x+i \varepsilon} \mathrm{d} x .
$$

One easily derives the distributive identities below by considering the logarithm in the complex plane, where $\log (z)=$ $\log |z|+i \operatorname{Arg}(z)$,

$$
\begin{aligned}
\frac{\mathrm{d}}{\mathrm{d} x} \log (x+i 0) & =\frac{1}{x+i 0}=\frac{\mathrm{d}}{\mathrm{d} x} \log (|x|)+\frac{\mathrm{d}}{\mathrm{d} x}(i \pi \Theta(-x)) \\
& =P \frac{1}{x}-i \pi \delta(x)
\end{aligned}
$$

Differentiating $n$ times leads to

$$
\begin{aligned}
\frac{\mathrm{d}}{\mathrm{d} x} \frac{1}{x+i \epsilon} & =-\frac{1}{(x+i \epsilon)^{2}}, \\
\frac{\mathrm{d}^{2}}{\mathrm{~d} x^{2}} \frac{1}{x+i \epsilon} & =+\frac{2}{(x+i \epsilon)^{3}}, \ldots, \frac{\mathrm{d}^{n}}{\mathrm{~d} x^{n}} \frac{1}{x+i \epsilon} \\
& =(-1)^{n} \frac{n !}{(x+i \epsilon)^{n+1}},
\end{aligned}
$$

therefore

$\frac{1}{(x+i 0)^{n+1}}=P \frac{1}{x^{n+1}}-(-1)^{n} \frac{i \pi}{n !} \delta^{\{n\}}(x)$,

where $\delta^{\{n\}}(x)$ denotes the $n$-fold derivative of $\delta(x)$ here, not the $n$-dimensional Dirac distribution often used in the paper.

\section{A.6 An important remark}

A multiplication of tempered distributions which is commutative and associative cannot be defined in general. One has

$$
(x \delta(x)) P \frac{1}{x}=0 P \frac{1}{x}=0 \neq \delta(x)\left(x P \frac{1}{x}\right)=\delta(x) .
$$

Unfortunately, distribution theory is linear. This is the origin of ultraviolet divergences in perturbative QFT. The problem may be illustrated by an analogy where one considers the Heaviside- $\Theta$ - and Dirac- $\delta$-distributions in one-dimensional 'configuration space'. The product of these two distributions, $\Theta(x) \delta(x)$, is obviously ill-defined; however, the distributional Fourier transforms

$$
\sqrt{2 \pi} \mathcal{F}\{\delta\}(k)=\sqrt{2 \pi} \hat{\delta}(k)=\int_{\mathbb{R}} \mathrm{d} x \delta(x) \mathrm{e}^{-i k x}=1,
$$

$$
\begin{aligned}
\sqrt{2 \pi} \hat{\Theta}(k) & =\lim _{\epsilon \searrow 0} \int_{\mathbb{R}} \mathrm{d} x \Theta(x) \mathrm{e}^{-i k x-\epsilon x} \\
& =\left.\lim _{\epsilon \searrow 0} \frac{i e^{-i k x-\epsilon x}}{k-i \epsilon}\right|_{0} ^{\infty}=-\frac{i}{k-i 0},
\end{aligned}
$$

exist and one may attempt to calculate the ill-defined product in 'momentum space', which formally goes over into a convolution,

$$
\begin{aligned}
& \sqrt{2 \pi} \mathcal{F}\{\Theta \delta\}(k) \\
& =\int_{\mathbb{R}} \mathrm{d} x \mathrm{e}^{-i k x} \Theta(x) \delta(x) \\
& =\int_{\mathbb{R}} \mathrm{d} x \mathrm{e}^{-i k x} \int_{\mathbb{R}} \frac{\mathrm{d} k^{\prime}}{\sqrt{2 \pi}} \hat{\Theta}\left(k^{\prime}\right) \mathrm{e}^{+i k^{\prime} x} \int_{\mathbb{R}} \frac{\mathrm{d} k^{\prime \prime}}{\sqrt{2 \pi}} \hat{\delta}\left(k^{\prime \prime}\right) \mathrm{e}^{+i k^{\prime \prime} x} .
\end{aligned}
$$

Since $\int_{\mathbb{R}} \mathrm{d} x \mathrm{e}^{i\left(k^{\prime}+k^{\prime \prime}-k\right) x}=2 \pi \delta\left(k^{\prime}+k^{\prime \prime}-k\right)$, one obtains

$$
\sqrt{2 \pi} \mathcal{F}\{\Theta \delta\}(k)=\int_{\mathbb{R}} \mathrm{d} k^{\prime} \hat{\Theta}\left(k^{\prime}\right) \hat{\delta}\left(k-k^{\prime}\right)=-\frac{i}{2 \pi} \int_{\mathbb{R}} \frac{\mathrm{d} k^{\prime}}{k^{\prime}-i 0} .
$$

The obvious problem in $\mathrm{x}$-space leads to a 'logarithmic UV divergence' in k-space. A concise description of the scaling properties of distributions, related to the wide-spread notion of power counting and the superficial degree of divergence of Feynman integrals, is crucial for the correct treatment of singular products of distributions in perturbative QFT. There, the rôle of the Heaviside $\Theta$-distribution is taken over by the time-ordering operator. The well-known textbook expression 
for the perturbative $S$-matrix given by

$$
\begin{aligned}
S & =\sum_{n=0}^{\infty} \frac{(-i)^{n}}{n !} \int_{-\infty}^{+\infty} \mathrm{d} t_{1} \ldots \int_{-\infty}^{+\infty} \mathrm{d} t_{n} T\left[H_{\text {int }}\left(t_{1}\right) \ldots H_{\text {int }}\left(t_{n}\right)\right] \\
& =\sum_{n=0}^{\infty} \frac{(-i)^{n}}{n !} \int_{\mathbb{R}^{4}} \mathrm{~d}^{4} x_{1} \ldots \int_{\mathbb{R}^{4}} \mathrm{~d}^{4} x_{n} T\left[\mathcal{H}_{\text {int }}\left(x_{1}\right) \ldots \mathcal{H}_{\text {int }}\left(x_{n}\right)\right],
\end{aligned}
$$

where the interaction Hamiltonian $H_{\text {int }}(t)$ is given by the interaction Hamiltonian density $\mathcal{H}_{\text {int }}(x)$ via

$H_{\text {int }}(t)=\int \mathrm{d}^{3} x \mathcal{H}_{\text {int }}(x)$

is problematic in the UV regime (and in the infrared regime, when massless fields are involved). A time-ordered expression à la

$$
\begin{aligned}
T & {\left[\mathcal{H}_{\text {int }}\left(x_{1}\right) \ldots \mathcal{H}_{\text {int }}\left(x_{n}\right)\right] } \\
& =\sum_{\substack{\text { Perm. } \Pi \\
\quad}} \Theta\left(x_{\Pi_{1}}^{0}-x_{\Pi_{2}}^{0}\right) \ldots \Theta\left(x_{\Pi_{(n-1)}}^{0}-x_{\Pi_{n}}^{0}\right) \\
& \mathcal{H}_{\text {int }}\left(x_{\Pi_{1}}\right) \ldots \mathcal{H}_{\text {int }}\left(x_{\Pi_{n}}\right)
\end{aligned}
$$

is formal (i.e., ill-defined), since the operator-valued distribution products of the $\mathcal{H}_{\text {int }}$ are simply too singular to be multiplied by $\Theta$-distributions.

\section{Appendix B: Asymptotic behavior of $d(x)$}

The symbol $\sim$ will be used in the following for asymptotic approximations, i.e. $f(x) \sim \Phi(x)$ if $f(x) / \Phi(x)$ tends to unity for $\mathbb{R} \ni x \rightarrow+\infty$ according to Landau [19]. Then $f$ is asymptotic to $\Phi$, or $\Phi$ is an asymptotic approximation to $f$.

From the well-known identities for (double or odd) factorials

$(2 n) !=(2 n) ! !(2 n-1) ! !, \quad(2 n) ! !=2^{n} n !$

and

$(2 n-1) ! !=\frac{2^{n}}{\sqrt{\pi}} \Gamma\left(n+\frac{1}{2}\right)=\frac{2^{n}}{\sqrt{\pi}}\left(n-\frac{1}{2}\right) !$

one readily obtains from

$\left(n-\frac{1}{2}\right) ! \sim \frac{n !}{\sqrt{n}}$

the asymptotic approximation

$(2 n) ! \sim \frac{2^{2 n}}{\sqrt{\pi}} \frac{(n !)^{2}}{\sqrt{n}}$.
Using Stirling's formula, this result can be generalized to

$$
\begin{aligned}
& (3 n) ! \sim \sqrt{2 \pi(3 n)}\left(\frac{3 n}{e}\right)^{3 n} \\
& =\frac{\sqrt{3} 3^{3 n}}{2 \pi n}\left[\sqrt{2 \pi n}\left(\frac{n}{e}\right)^{n}\right]^{3} \sim \frac{\sqrt{3} 3^{3 n}}{2 \pi n}(n !)^{3} .
\end{aligned}
$$

Accordingly, $d(x)$ in Eq. (102) can be approximated by

$$
\begin{aligned}
d(x) & =\sum_{n=2}^{\infty} \frac{x^{n}}{n !(n-1) !(n-2) !}=\sum_{n=2}^{\infty} \frac{n^{2}(n-1)}{(n !)^{3}} x^{n} \\
& \sim \sum_{n=0}^{\infty} \frac{n^{3}}{(n !)^{3}} x^{n} \sim \frac{\sqrt{3}}{2 \pi} \sum_{n=0}^{\infty} \frac{3^{3 n} n^{2}}{(3 n) !} x^{n}
\end{aligned}
$$

or

$d(x) \sim \frac{\sqrt{3}}{2 \pi} \sum_{n=0}^{\infty} \frac{n^{2}}{(3 n) !}\left(3 x^{1 / 3}\right)^{3 n}$.

A straightforward but rather tedious calculation shows that

$$
\begin{aligned}
\sum_{n=0}^{\infty} & \frac{n^{2}}{(3 n) !} y^{3 n} \\
= & \frac{1}{27} y(y+1) e^{y}-\frac{y}{27} \mathrm{e}^{-\frac{y}{2}}\left[(y+1) \cos \left(\frac{\sqrt{3} y}{2}\right)\right. \\
& \left.-\sqrt{3}(y-1) \sin \left(\frac{\sqrt{3} y}{2}\right)\right] .
\end{aligned}
$$

Note that the sine term contains an additional factor $\sqrt{3}$, which is missing in the cosine term. Keeping only the dominant term, one has asymptotically

$\sum_{n=0}^{\infty} \frac{n^{2}}{(3 n) !} y^{3 n} \sim \frac{y^{2}}{27} e^{y}$

and setting $y=3 x^{1 / 3}$ leads to the desired result:

$d(x) \sim \frac{\sqrt{3}}{2 \pi} \frac{\left(3 x^{1 / 3}\right)^{2}}{27} \mathrm{e}^{3 x^{1 / 3}}=\frac{1}{2 \pi \sqrt{3}} x^{2 / 3} \mathrm{e}^{3 x^{1 / 3}}$.

\section{References}

1. R.F. Streater, A.S. Wightman, PCT, Spin, Statistics and All That (Benjamin-Cummings Publishing Company, San Francisco, 1964)

2. E. Wigner, On the unitary representations of the inhomogeneous Lorentz group. Ann. Math. 40, 149-204 (1939)

3. D. Buchholz, Gauss' law and the infraparticle problem. Phys. Lett. B 174, 331-334 (1986)

4. B. Schroer, Infrateilchen in der Quantenfeldtheorie. Fort. der Physik 11, 1-32 (1963)

5. A. Wintner, The unboundedness of quantum-mechanical matrices. Phys. Rev. 71, 738-739 (1947)

6. H. Wielandt, Über die Unbeschränktheit der Operatoren der Quantenmechanik. Math. Ann. 121, 21-21 (1949) 
7. F. Constantinescu, Distributionen und ihre Anwendungen in der Physik (B. G. Teubner, Stuttgart, 1974)

8. B. Schroer, The concept of nonlocalizable fields and its connection with nonrenormalizable field theories. J. Math. Phys. 5(10), 13611367 (1964)

9. A. Jaffe, High-energy behavior in quantum field theory. I. Strictly localizable fields. Phys. Rev. 158(5), 1454-1461 (1967)

10. F. Krahe, Causal perturbation theory for massive vector Boson theories. Acta Phys. Pol. B 27, 2453-2476 (1996)

11. A.V. Razumov, G.N. Rybkin, State space in BRST-quantization of gauge-invariant systems. Nucl. Phys. B 332, 209-223 (1990)

12. J. Bognar, Indefinite Inner Product Spaces (Springer, Berlin, 1974)

13. W. Massey, Homology and Cohomology Theory (Dekker, New York, 1978)
14. T. Kugo, I. Ojima, Local covariant operator formalism of nonabelian gauge theories and quark confinement problem. Suppl. Prog. Theor. Phys. 66, 1-130 (1979)

15. H. Araki, On the connection of spin and commutation relations between different fields. J. Math. Phys. 2, 267-270 (1961)

16. M. Dütsch, T. Hurth, G. Scharf, Causal construction of Yang-Mills theories. 4. Unitarity. Nuovo Cim. A 108, 737-774 (1995)

17. J.C. Ward, An identity in quantum electrodynamics. Phys. Rev. 78, 182-182 (1950)

18. A. Aste, G. Scharf, Non-Abelian gauge theories as a consequence of perturbative quantum gauge invariance. Int. J. Mod. Phys. A 14, 3421-3432 (1999)

19. E. Landau, Vorlesungen über Zahlentheorie (Hirzel, Stuttgart, 1927) 